\title{
Geochemical hunting of lithogenic and anthropogenic impacts on polymetallic distribution (Bregalnica river basin, Republic of Macedonia)
}

\author{
Biljana Balabanova, Trajče Stafilov, Robert Šajn \& Claudiu Tănăselia
}

To cite this article: Biljana Balabanova, Trajče Stafilov, Robert Šajn \& Claudiu Tănăselia (2016) Geochemical hunting of lithogenic and anthropogenic impacts on polymetallic distribution (Bregalnica river basin, Republic of Macedonia), Journal of Environmental Science and Health, Part A, 51:13, 1180-1194, DOI: 10.1080/10934529.2016.1206389

To link to this article: http://dx.doi.org/10.1080/10934529.2016.1206389

曲 Published online: 26 Jul 2016.

Submit your article to this journal ¿

ЦIl Article views: 5

View related articles $\sqsubset$

View Crossmark data $\nearrow$ 


\title{
Geochemical hunting of lithogenic and anthropogenic impacts on polymetallic distribution (Bregalnica river basin, Republic of Macedonia)
}

\author{
Biljana Balabanova ${ }^{a}$, Trajče Stafilov ${ }^{b}$, Robert Šajnc, and Claudiu Tănăselia ${ }^{d}$ \\ aFaculty of Agriculture, University "Goce Delčev", Štip, Republic of Macedonia; 'Institute of Chemistry, Faculty of Science, Ss. Cyril and Methodius \\ University, Macedonia; 'Geological Survey of Slovenia, Ljubljana, Slovenia; ${ }^{d}$ INCDO-INOE 2000 Research Institute for Analytical Instrumentation (ICIA), \\ Cluj-Napoca, Romania
}

\begin{abstract}
The main subject of this investigation was the assessment of the lithogenic and anthropogenic distribution of 69 elements in the sediments and fluvisol in the Bregalnica river basin. Alluvial soil and fluvisol samples were collected from the total of eighteen locations along the course of the Bregalnica river and additional thirteen samples were collected from its tributaries. The matrix elements accumulation patterns followed the order: $\mathrm{Fe}>\mathrm{Na}>\mathrm{Al}>\mathrm{Ca}>\mathrm{Mg}>\mathrm{K}>\mathrm{Ti}>\mathrm{P}$. The potentially toxic elements, such as $\mathrm{As}, \mathrm{Cd}, \mathrm{Pb}$, and $\mathrm{Zn}$, have enriched content in the sediments in the medium course of the river, where the main anthropogenic introduction activities occurred. By multivariate analysis the dominant geochemical associations were extracted, as follows: F1: Y-Eu-Lu-Cr-V-La-Gd-Nb-Co-Hf-Zr-Ga-Mg-Fe-Sr-Ta-Sn-Li-Na-Rb$\mathrm{Ni}-\mathrm{Ge}-\mathrm{Be}-\mathrm{Cs}$; F2: As-W-Ba-Ag-Cu-TI-Zn-Sb-Mo-In-Cd-Te-Bi-Pb and F3: I-Sc-Br-K. Lead and zinc contents were strongly correlated with the hydrothermal exploitations, especially in the area of Neogene clastite and vulcanite. These elements occur as dominant geochemical markers of the anthropogenic impacts of polymetallic enrichments due to the hydrothermal ore exploitation (Factor 2). The impact of Oligocene volcanism (Kratovo-Zletovo region) was observed in the lithological enrichments of $\mathrm{Pb}, \mathrm{Zn}, \mathrm{Cu}, \mathrm{As}, \mathrm{Sb}, \mathrm{Mo}$ and $\mathrm{Bi}$. Despite the natural distribution along the course of the Bregalnica river, an exceptional anomaly in the iron distribution of the old polymetallic unused mineralization was detected in Zone 1 (Berovo region).
\end{abstract}

ARTICLE HISTORY

Received 7 May 2016

\section{KEYWORDS}

Alluvial soil; metals; pollution; multivariate analysis; spatial distribution

\section{Introduction}

Alluvial soils have been used as an important tool to assess the health status of aquatic ecosystems and represent an integral component of ecological integrity. ${ }^{[1-4]}$ Sediments act as a sink of inorganic pollutants (potentially toxic elements/metals) and provide a history of anthropogenic pollutant input. ${ }^{[5]}$ Toxic metals pollution in sediments has been proved to be an increasingly global problem, which is considered to pose a serious threat to the aquatic environment resulting from their toxicity, non-biodegradable and persistent nature, and the bio-enrichment ability in food chain. ${ }^{[4,6-9]}$ These elements are deposited onto sediment surfaces and immobilized through adsorption, coagulation or flocculation and incorporation into the lattice structure of minerals and precipitation by forming insoluble fractionation. ${ }^{[4,10,11]}$ Only a small portion of free metal ions, however, stay dissolved in water. ${ }^{[12]}$ Thus, the distribution of trace metals in sediment adjacent to populated areas (includes industries, mining exploitation in particular) can give us the evidence of anthropogenic influence on aquatic system and convenience in assessing the potential risks associated with human waste discharge. ${ }^{[13]}$ For example, in the Republic of Macedonia there are several significant pollution sources of potentially toxic elements/metals that continuously introduce tailings and waste into river waters. ${ }^{[14]}$ These hotspots are predominantly present in the southeast part of the country. On the other hand, this region is characterized by a dominance of very old geological units specific for the Balkans area and even Europe as a whole. Regarding the dominance of the volcanic geological units and $\mathrm{Pb}-\mathrm{Zn}$ mineral deposits, the region of the Bregalnica river basin is a specific region to monitor the geochemical interactions in various alluvial soils. ${ }^{[14,15]}$ This region is characterized by several significant pollution sources of potentially toxic metals and other chemical elements in the environment: the copper mine and flotation Bučim near Radoviš and the $\mathrm{Pb}-\mathrm{Zn}$ mines Sasa near Makedonska Kamenica and Zletovo near Probištip. ${ }^{[16]}$ The excavation of the copper minerals is carried out from an open ore pit, while in the leadzinc mines the exploitation is underground, and the ore tailings are stored outdoors. The ore produced in the mines is processed in the flotation plants, and in the process of flotation of the relevant minerals, flotation tailings are separated and disposed on a dumpsite in the open air.

The spatial hunting of dominant elements associations in the Bregalnica river basin represents the first attempt of this type in this unique area. The presence of dominant Oligocene volcanism in the area of the Sasa mine ( $\mathrm{Pb}-\mathrm{Zn}$ hydrothermal exploitation), the Kratovo-Zletovo district and in the area of the Bučim mine ( $\mathrm{Cu}-\mathrm{Au}$ hydrothermal exploitation) creates specific

CONTACT Trajče Stafilov trajcest@pmf.ukim.mk E Institute of Chemistry, Faculty of Natural Sciences and Mathematics, Ss. Cyril and Methodius University, POB 162,1000 Skopje, Macedonia.

Color versions of one or more of the figures in this article can be found online at www.tandfonline.com/lesa.

(C) 2016 Taylor \& Francis Group, LLC 
environmental conditions leading to lithogenic and anthropogenic poly-metallic enrichments. Therefore, the main objective of this study was to assess the lithogenic and anthropogenic distribution of a total of 69 elements along the Bregalnica river basin.

Multivariate statistical approach was implemented for hunting the dominant geochemical associations. Alluvial soil samples (sediment and fluvisol) were collected for the historical record of the lithological and anthropological impact on the elements' geochemistry. The dominant lithogenic and anthropogenic markers were extracted and the spatial pattern of each geochemical marker was generated.

\section{Materials and methods}

\section{Investigated area}

The investigated area includes the basin of the river Bregalnica, which is located in the east planning region of the Republic of
Macedonia. The area of the Bregalnica river basin covers $\sim 200 \mathrm{~km}(\mathrm{~W}-\mathrm{E}) \times 200 \mathrm{~km}(\mathrm{~S}-\mathrm{N})$ within the following geographic coordinates $\mathrm{N}: 41^{\circ} 27^{\prime}-42^{\circ} 09^{\prime}$ and E: $22^{\circ} 55^{\prime}-23^{\circ} 01^{\prime}$ (Fig. 1). The region of the investigated area is geographically composed of several sub-regions. These sub-regions are characterized by specific generalized geology and land use. As a consequence of the industrialization, urbanization and lack of treatment of the wastewaters from the industry, the mines and the city sewerage, the waters of this important hydrographic factor are exposed to a high level of pollution, from the aspect of introduction of higher contents of certain toxic metals. The pollution is frequently particularly high at low river flows, as in the case of the river Bregalnica in its course through the Kočani valley (central part of the investigated area). The east and north parts of the investigated area are mainly mountainous parts while the central and western parts consist of cultivated agricultural land (Fig. 2).

The precipitation is mostly related to and conditioned by the Mediterranean cyclones. During the summer period, the region

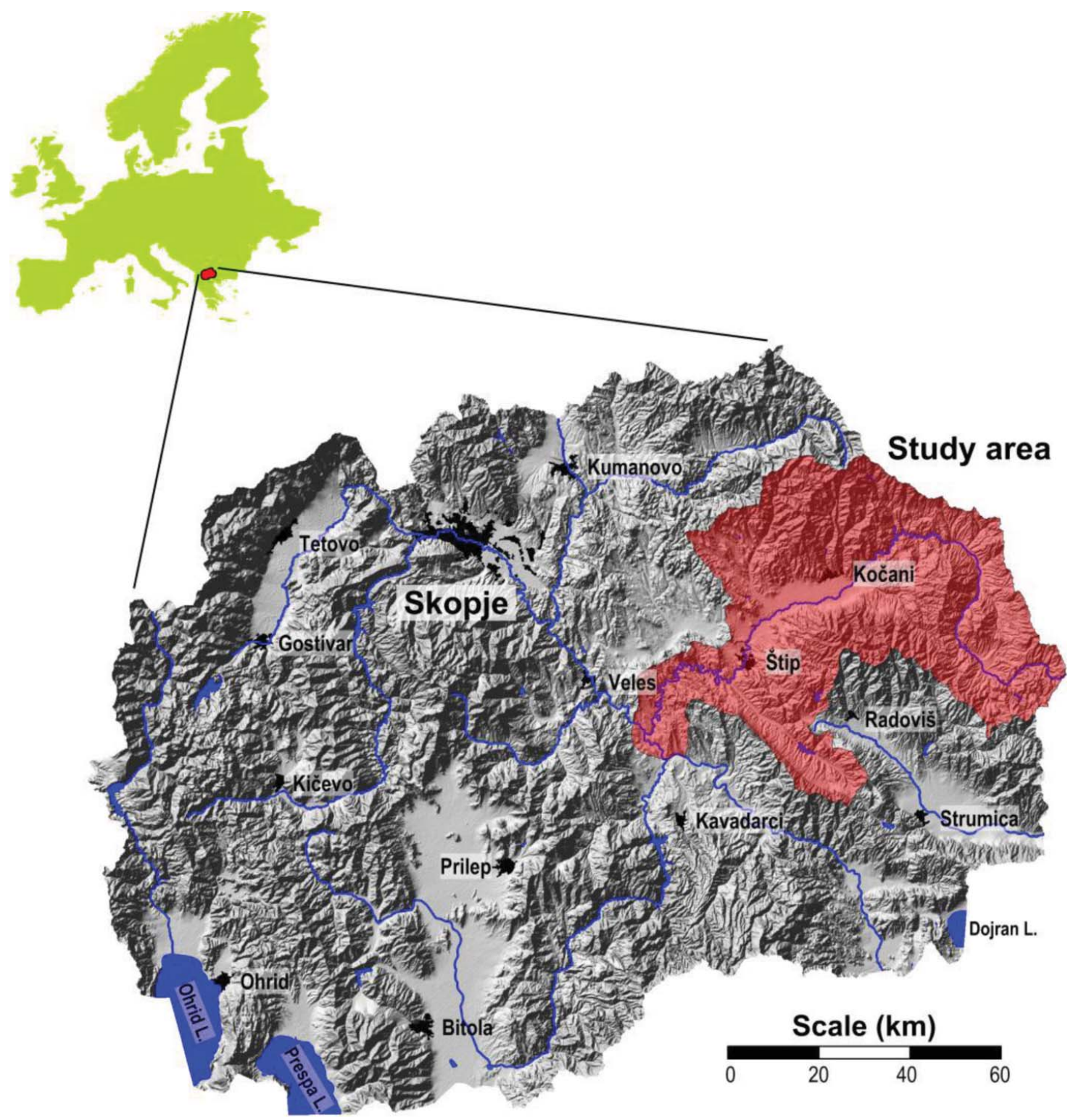

Figure 1. The investigated area on the territory of the Republic of Macedonia. 


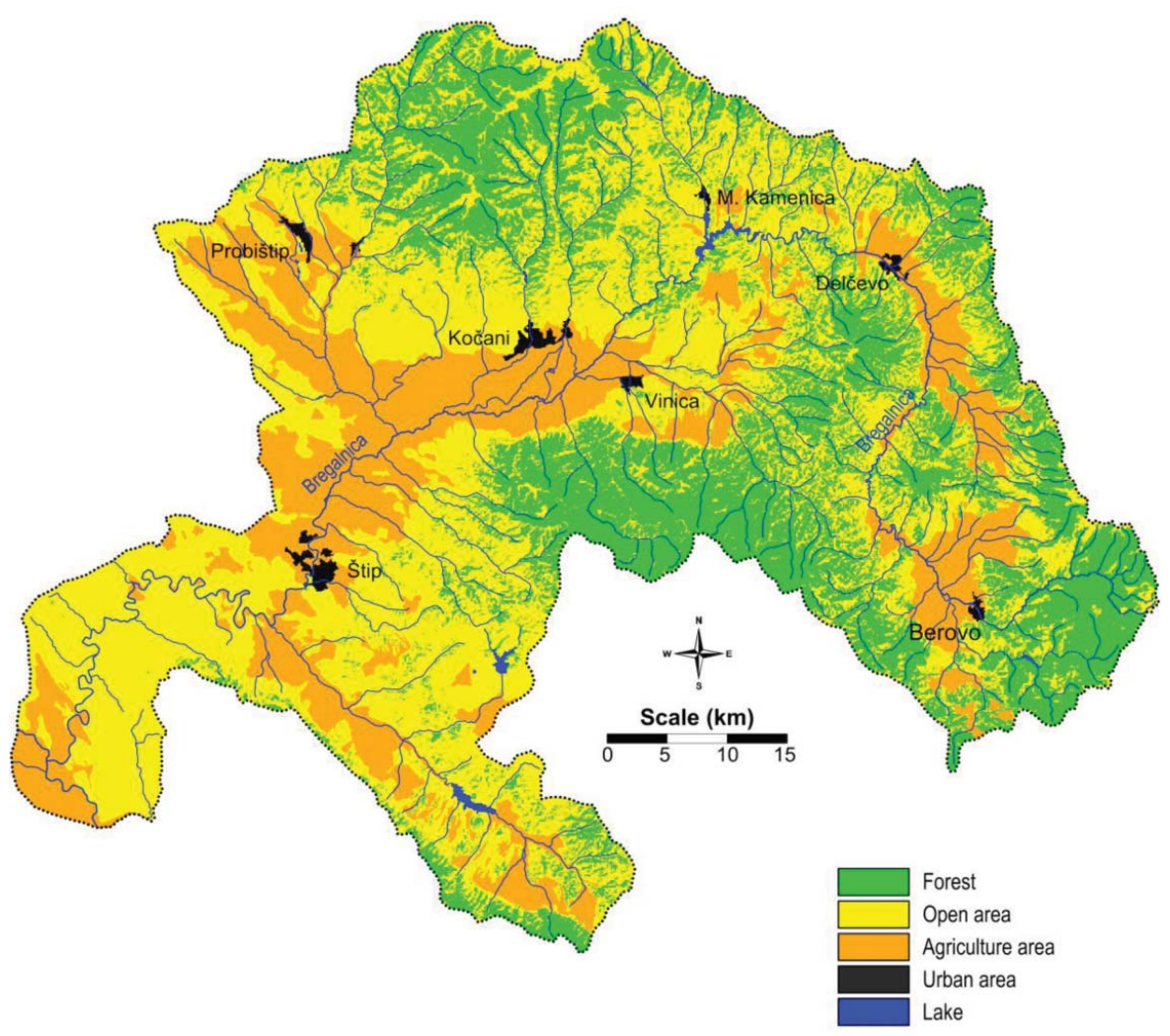

Figure 2. The region of the investigated area including the type of land use.

is most often found in the centre of the subtropical anticyclone, which causes warm and dry summers. From the central area of the region, as the driest area, the average annual precipitation increases in all directions, because of the increase either in the influence of the Mediterranean climate or the increase in altitude. As regards the total annual number of sunny hours, there are about $6 \mathrm{~h}$ per day in this area. In the region are distinguished about ten climatic-vegetation soil areas with considerably heterogeneous climate, soil and vegetation characteristics. ${ }^{[17]}$

\section{Generalized geology and dominant ore mineralization of the investigated area}

Southeast Europe has undergone a complex Alpine tectonic evolution. The Srednogorie zone, Kraishte and the Rhodope Massif in Serbia, Macedonia and Bulgaria were sites of extensive westward/southward younging magmatic activity, starting in the Jurassic and up to the Cretaceous period, with the closure of the Vardar zone. ${ }^{[18,19]}$ The investigated area that covers the basin of the river Bregalnica lies on two main tectonic units: the Serbian-Macedonian massive and the Vardar zone. ${ }^{[19]}$ The polyphasic Neogene deformations associated with the volcanic activities through the insignificant movements had direct influence on the gradual formation of the reefs and the formation of deposits in the existing basins (Fig. 3). From the middle Miocene to the end of the Pleistocene there were alternating periods of fast and slow landslide accompanied with variable sedimentation (deposition).

The Cenozoic volcanism represents a more recent extension in the Serbian-Macedonian massive and the Vardar zone.
The oldest volcanic rocks can be found in the areas of Bučim, Damjan, the Borov Dol district and in the zone of Toranica, Sasa, Delčevo and Pehčevo. ${ }^{[18]}$ These older volcanic rocks were formed in the mid Miocene from sedimentary rocks that represent the upper age limit of the rocks. The origin of these oldest volcanic rocks is related to the Oligocene - the early Miocene period. The following are categorized as volcanic rocks: andesite, latite, quartz-latite and dacite. Volcanism appears sequentially and in several phases forming sub-volcanic areas. On the other hand, the pyroclastites are most frequently found in the Kratovo-Zletovo volcanic area, where the dacites and andesites are the oldest formations. Generalized geology of the area is generated based on the data provided by Rakicević et al. and presented in Figure 3. ${ }^{[20]}$ The most important Macedonian metal deposits are related to the regional magmatic activity that occurred in the southern parts of the Carpato-Balkanides during the Eocene-Pliocene. ${ }^{[21,22]}$ The Zletovo mine is located near the town of Probištip. As presented by Serafimovski et al. the mineralization of this deposit is related to the Tertiary calc-alkaline magmatic rocks (dacites and andesites) and it is found in a dacitic volcano-sedimentary suite that had been altered to clays and micas. ${ }^{[21]}$ The Sasa mine is situated near the city of Kamenica. The mineralization is located along the contact line between the Miocene calcalkaline igneous bodies (latites and dacites) and the graphitechlorite-sericite shists, gneisses and limestones. ${ }^{[23]}$ The ore consists of pyrite, galena and sphalerite with additional magnetite and chalcopyrite, while the mass fractions of $\mathrm{Pb}$ and $\mathrm{Zn}$ are around $10 \%$ with additional contents of $\mathrm{Ag}, \mathrm{As}, \mathrm{Cd}, \mathrm{Mn}$ and $\mathrm{Sb}$. The ore is concentrated at the mine by flotation and the tailings are stored in a dam in the valley just below the 


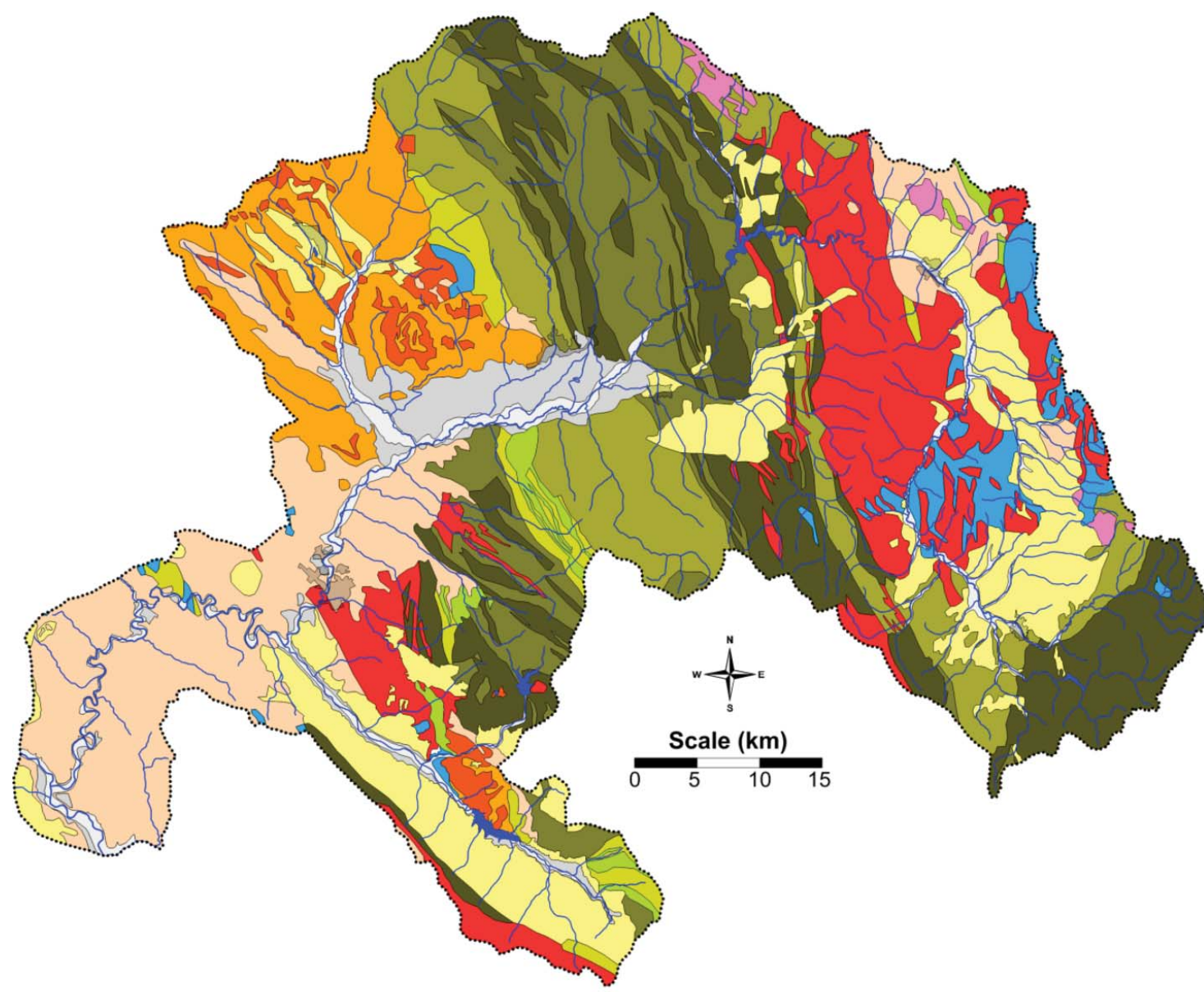

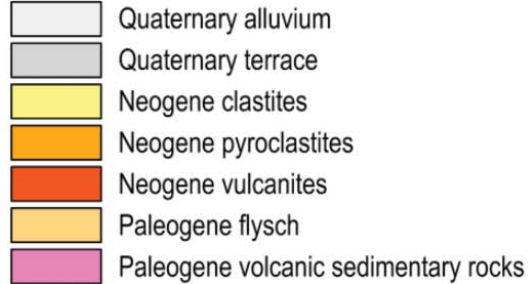

Figure 3. Generalized geology of the whole investigated area.

mine. The Kamenička River is culverted beneath the tailings dam and flows for $12 \mathrm{~km}$ until it meets in the Kalimanci Lake (artificial reservoir on the Bregalnica River). The Bucim mine is situated near the town of Radoviš. Copper-porphyry type of mineralization occurs in this deposit. ${ }^{[23,24]}$ The ore concentrate is characterized by average content of $0.3 \% \mathrm{Cu}$ and $0.3 \mathrm{~g} / \mathrm{t}$ $\mathrm{Au}^{[25]}$ The milling and flotation plants discharge their drainage waters in the river Lakavica.

\section{Sampling strategy and protocols}

In the period August-November 2012, samples of river sediments and fluvisol were collected, as well as natural and anthropogenic alluvium from the river plains along the course of the river Bregalnica, in accordance with a previously specified sampling network. Apart from the above-mentioned locations for sample collection along the river course, some of the more important tributaries to the river Bregalnica were also additionally included in the location network, in order to determine the influence of the potentially polluted waters from the tributaries on the pollution of the main hydrographic factor. The more important tributaries of the river Bregalnica that were included in the sample network were the following: Ratevska River, Očipalska River, Kamenička River, Kočanska River, Orizarska River, Zletovska River, the river Lakavica and Ovčepolska River. Samples of water and river sediment were also collected from the river Vardar in the area where the river Bregalnica flows into the river Vardar.

The total basin area of the river Bregalnica was divided into four zones: zone 1 (Z-1) which includes 5 locations (B1, B2, B3, B4, and P1), zone 2 (Z-2), which includes the following 5 locations: B5, B6, B7, B8 and P2, zone 3 (Z-3) which includes the following 5 locations: B9, B10, B11, $\mathrm{B} 12$, and $\mathrm{B} 13$ and zone $4(\mathrm{Z}-4)$ which includes the following locations: B14, B15, B16, B17 and B18. The areas of the Kamenička River $(\mathrm{K})$, Zletovska River $(\mathrm{Z})$ and the river 


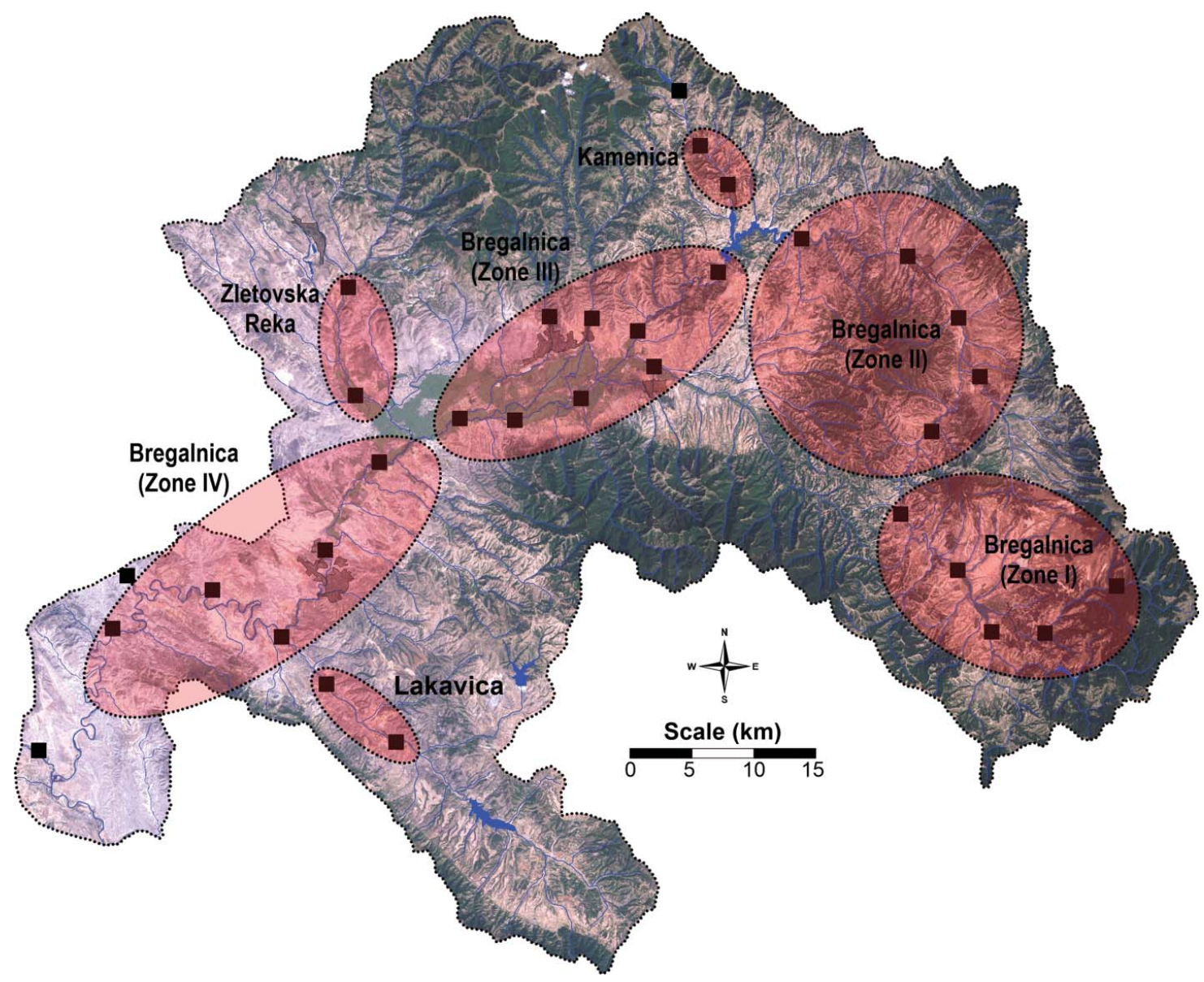

Figure 4. Locations for taking samples of water and sediment along the course of the river Bregalnica.

Lakavica (L) were separately monitored (Fig. 4). According to the previously set of sampling strategy, at each location sediment samples were collected using the following protocol: Fine grained sediment $(0.06-2.0 \mathrm{~mm})$ were carefully collected from the top two to four centimeters as a surface sediment composite sample ( $2-3$ spots from one side of the river). Sediment was collected from beneath an aqueous layer directly, using plastic shovel. Following collection, sediment was transferred from the sampling device to a sample container for the analyses requested. Multiple grabs were placed into a container constructed of inert material, compositing representative sample. At the same location, wherefrom the sediments were collected, fluvisol samples were also collected from the alluvial terraces. The top layer $(0-5 \mathrm{~cm})$ of fluvisol was collected using plastic shovel. The representative sample was constructed collecting the fluvisol from the corners of 1 meter square.

Along the whole course of the river Bregalnica a total of 18 samples of sediments were collected. Additionally, samples were also collected from another 13 locations along the tributaries to the Bregalnica. The locations wherefrom the samples were collected are presented in Figure 4. The geographic coordinates of each location were also determined to enable mapping of the generated information about the values of the contents of the investigated elements. This step is nevertheless required for the monitoring of the distribution of the investigated elements along the course of the river Bregalnica.

\section{Sample physical-chemical pre-treatment}

The preparation of the collected samples for the analysis of the 69 elements was carried out by their cleaning, drying, breaking and digestion. For digestion of the samples of alluvial soil the method of open digestion with mixture of mineral concentrated acids was applied. The samples brought in the laboratory were subjected to cleaning and homogenization, drying at room temperature, or in a drying room at $40^{\circ} \mathrm{C}$, to a constantly dry mass. Then the samples were passed through a 2-mm sieve and finally were homogenized by grinding in a porcelain mortar until reaching a final size of the particles of $25 \mu \mathrm{m}$. Following the physical preparation, the samples were chemically prepared by wet digestion, applying a mixture of acids in accordance with the international standard. ${ }^{[26]}$ Precisely measured mass of sample $(0.25 \mathrm{~g})$ was placed in Teflon vessels and $5 \mathrm{~mL}$ concentrated nitric acid $\left(\mathrm{HNO}_{3}, 69 \%\right.$, wt $\mathrm{v}^{-1}$, trace select purity, Merck, Munich, Germany) was added, until the brown vapors came out of the vessels, on reaching a temperature of $180^{\circ} \mathrm{C}$. For total digestion of inorganic components, $5-10 \mathrm{~mL}$ of hydrofluoric acid (HF, $\geq 40 \%$, wt $\mathrm{v}^{-1}$ p.a. Merck, Munich, Germany) was added. When the digest became a clear solution, $2 \mathrm{~mL}$ of perchloric acid $\left(\mathrm{HClO}_{4}\right.$, p.a. purity, Alkaloid, Skopje, Macedonia) was added for total digestion of the organic compounds. After cooling the vessels for $15 \mathrm{~min}, 2 \mathrm{~mL}$ of hydrochloric acid $\left(\mathrm{HCl}, 37 \%\right.$, $\mathrm{t}, \mathrm{wt}^{-1}$, trace select purity, Merck, Munich, Germany) and $5 \mathrm{~mL}$ of double ionized water were added for dissolving of metal ions totally. Finally, the digest was transferred into the $25 \mathrm{~mL}$ volumetric flasks. Thus, the digested alluvial soil samples 
were prepared for determination of the contents of the different elements by applying atomic emission and mass spectrometry.

\section{Instrumentation and determination of the elements content}

SCIEX Perkin Elmer Elan DRC II (Canada) inductively coupled plasma mass spectrometer (quadrupole as single detector) was

Table 1. Instrumental conditions for ICP-MS and ICP-AES.

\begin{tabular}{|c|c|c|}
\hline RF Generator & ICP-AES & ICP-MS \\
\hline $\begin{array}{l}\text { Power output of RF } \\
\text { generator }\end{array}$ & $1500 \mathrm{~W}$ & \\
\hline $\begin{array}{l}\text { Power output } \\
\text { stability }\end{array}$ & Better than $0.1 \%$ & \\
\hline ICP Ar flow gas rate & $15 \mathrm{~L} \mathrm{~min}^{-1}$ & \\
\hline \multicolumn{3}{|l|}{ Plasma parameters } \\
\hline Nebulizer & V-groove & Micromist \\
\hline Spray chamber & Double-pass cyclone & \\
\hline Peristaltic pump & $0-50 \mathrm{rpm}$ & \\
\hline Cones & / & Platinum \\
\hline $\begin{array}{l}\text { Plasma } \\
\text { configuration }\end{array}$ & Radially viewed & Axially viewed \\
\hline Spectrometer & Echelle optical design & Quadrupole \\
\hline Polychromator & $400 \mathrm{~mm}$ focal length & l \\
\hline $\begin{array}{l}\text { Polychromator } \\
\text { purge }\end{array}$ & $0.5 \mathrm{~L} \mathrm{~min}^{-1}$ & l \\
\hline Total voltage/ $\mathrm{V}$ & / & 0.1 \\
\hline $\begin{array}{l}\text { Integration } \\
\text { measurement } \\
\text { time/ms }\end{array}$ & / & 0.1 \\
\hline $\begin{array}{l}\text { Measurement at } \\
\text { one point } \\
\text { (isotope)/s }\end{array}$ & / & 300 \\
\hline $\begin{array}{l}\text { Repetitions } \\
\text { measurement }\end{array}$ & 3 per point & \\
\hline \multicolumn{3}{|l|}{$\begin{array}{l}\text { Conditions for } \\
\text { program }\end{array}$} \\
\hline $\begin{array}{l}\text { ICP-AES } \\
\text { measurements }\end{array}$ & ICP-MS measurements & \\
\hline Element & Wavelength, nm & Isotopes \\
\hline Al & 396.152 & $107 \mathrm{Ag}, 75 \mathrm{As}, 27 \mathrm{Al}$ \\
\hline $\mathrm{Ca}$ & 370.602 & 197Au, 11B, \\
\hline $\mathrm{Fe}$ & 238.204 & 137Ba, 9Be, \\
\hline $\mathrm{Mg}$ & 280.270 & 209Bi, 79Br, \\
\hline $\mathrm{K}$ & 766.491 & $114 \mathrm{Cd}, 140 \mathrm{Ce}$ \\
\hline $\mathrm{Na}$ & 589.592 & $59 \mathrm{Co}, 53 \mathrm{Cr}$ \\
\hline$P$ & 213.618 & $\begin{array}{l}\text { 133Cs, 63Cu, } \\
\text { 163Dy, 166Er, } \\
\text { 153Eu, 56/57Fe, } \\
69 \mathrm{Ga}, 157 \mathrm{Gd}, \\
72 \mathrm{Ge}, 178 \mathrm{Hf}, 201 / \\
202 \mathrm{Hg}, 165 \mathrm{Ho}, \\
127 \mathrm{I}, 115 \mathrm{In}, 193 \mathrm{Ir}, \\
\text { 139La, 7Li, 175Lu, } \\
55 \mathrm{Mn}, 95 \mathrm{Mo}, \\
93 \mathrm{Nb}, 146 \mathrm{Nd}, \\
60 \mathrm{Ni}, 189 \mathrm{Os}, 206 / \\
207 / 208 \mathrm{~Pb}, \\
105 \mathrm{Pd}, 141 \mathrm{Pr}, \\
195 \mathrm{Pt}, 85 \mathrm{Rb}, \\
185 \mathrm{Re}, 103 \mathrm{Rh}, \\
101 \mathrm{Ru}, 121 \mathrm{Sb}, \\
45 \mathrm{Sc}, 77 \mathrm{Se}, \\
147 \mathrm{Sm}, 120 \mathrm{Sn}, \\
88 \mathrm{Sr}, 181 \mathrm{Ta}, \\
159 \mathrm{~Tb}, 125 \mathrm{Te}, \\
47 \mathrm{Ti}, 232 \mathrm{Th}, \\
205 \mathrm{Tl}, 169 \mathrm{Tm}, \\
51 \mathrm{~V}, 182 \mathrm{~W}, 89 \mathrm{Y}, \\
172 \mathrm{Yb}, 66 \mathrm{Zn}, \\
90 \mathrm{Zr}\end{array}$ \\
\hline
\end{tabular}

used for measurement of the concentration of the trace elements. All measurements were carried out using the semiquantitative method (Total Quant) supplied by Elan 3.4 software that uses a response factor calibration curve, which was obtained by calibration in multiple points, low, medium, and high concentration, for optimum setup, using a multielement Merck VI standard solution, diluted to mimic the real sample consumption. Atomic emission spectrometer with inductively coupled plasma, ICPAES, (model 715ES, Varian, Palo Alto, CA, USA), was used for measurement of the concentration of several elements ( $\mathrm{Al}, \mathrm{Ca}$, $\mathrm{Fe}, \mathrm{K}, \mathrm{Mg}, \mathrm{Na}$ and $\mathrm{P}$ ). For each element analyzed, previous optimization of the instrumental conditions was performed. The instrumental and operating conditions for each of the abovementioned techniques are presented in Table 1 . In all samples, the contents of a total of 69 elements were analyzed: $\mathrm{Ag}, \mathrm{As}, \mathrm{Al}$, $\mathrm{Au}, \mathrm{B}, \mathrm{Ba}, \mathrm{Be}, \mathrm{Bi}, \mathrm{Br}, \mathrm{Ca}, \mathrm{Cd}, \mathrm{Ce}, \mathrm{Co}, \mathrm{Cr}, \mathrm{Cs}, \mathrm{Cu}, \mathrm{Dy}$, Er, Eu, Fe, $\mathrm{Ga}, \mathrm{Gd}, \mathrm{Ge}, \mathrm{Hf}, \mathrm{Hg}$, Ho, I, In, Ir, K, La, Li, Lu, Mg, Mn, Mo, Na, $\mathrm{Nb}, \mathrm{Nd}, \mathrm{Ni}$, Os, P, Pb, Pd, Pr, Pt, Rb, Re, Rh, Ru, Sb, Sc, Se, Sm, Sn, Sr, Ta, Tb, Te, Ti, Th, Tl, Tm, V, W, Y, Yb, Zn and Zr.

The limits of detection (LOD) were based on the usual definition as the concentration of the analytic yielding a signal equivalent to three times the standard deviation of the blank signal, using 10 measurements of the blank for this calculation. The calculated values for the detection limits are presented in Table 2. Both certified reference materials (NIST-SRM 2711a, Montana II Soil, National Institute of Standards \& Technologies, Gaithersburg, MD, USA) and spiked intra-laboratory

Table 2. Lower detection limit for the analyzed elements.

\begin{tabular}{|c|c|c|c|c|c|}
\hline Element & Unit & $\mathrm{LOD}^{\mathrm{a}}$ & Element & Unit & $\operatorname{LOD}^{a}$ \\
\hline $\mathrm{Ag}$ & $\mathrm{mg} / \mathrm{kg}$ & 0.01 & $\mathrm{Mn}$ & $\mathrm{mg} / \mathrm{kg}$ & 0.01 \\
\hline $\mathrm{Al}$ & $\%$ & 0.9 & Mo & $\mathrm{mg} / \mathrm{kg}$ & 0.01 \\
\hline As & $\mathrm{mg} / \mathrm{kg}$ & 0.1 & $\mathrm{Na}$ & $\%$ & 0.1 \\
\hline $\mathrm{Au}$ & $\mathrm{mg} / \mathrm{kg}$ & 0.01 & $\mathrm{Nb}$ & $\mathrm{mg} / \mathrm{kg}$ & 0.1 \\
\hline B & $\mathrm{mg} / \mathrm{kg}$ & 0.01 & $\mathrm{Nd}$ & $\mathrm{mg} / \mathrm{kg}$ & 0.01 \\
\hline $\mathrm{Ba}$ & $\mathrm{mg} / \mathrm{kg}$ & 0.01 & $\mathrm{Ni}$ & $\mathrm{mg} / \mathrm{kg}$ & 0.01 \\
\hline $\mathrm{Be}$ & $\mathrm{mg} / \mathrm{kg}$ & 0.01 & Os & $\mu \mathrm{g} / \mathrm{kg}$ & 0.1 \\
\hline $\mathrm{Bi}$ & $\mathrm{mg} / \mathrm{kg}$ & 0.01 & $P$ & $\mathrm{mg} / \mathrm{kg}$ & 10 \\
\hline $\mathrm{Br}$ & $\mathrm{mg} / \mathrm{kg}$ & 0.01 & $\mathrm{~Pb}$ & $\mathrm{mg} / \mathrm{kg}$ & 0.01 \\
\hline $\mathrm{Ca}$ & $\%$ & 10 & $\mathrm{Pd}$ & $\mathrm{mg} / \mathrm{kg}$ & 0.1 \\
\hline $\mathrm{Cd}$ & $\mathrm{mg} / \mathrm{kg}$ & 0.01 & $\operatorname{Pr}$ & $\mathrm{mg} / \mathrm{kg}$ & 0.01 \\
\hline $\mathrm{Ce}$ & $\mathrm{mg} / \mathrm{kg}$ & 0.05 & $\mathrm{Pt}$ & $\mathrm{mg} / \mathrm{kg}$ & 0.01 \\
\hline Co & $\mathrm{mg} / \mathrm{kg}$ & 0.001 & $\mathrm{Rb}$ & $\mathrm{mg} / \mathrm{kg}$ & 0.1 \\
\hline $\mathrm{Cr}$ & $\mathrm{mg} / \mathrm{kg}$ & 0.001 & $\mathrm{Re}$ & $\mathrm{mg} / \mathrm{kg}$ & 0.01 \\
\hline Cs & $\mathrm{mg} / \mathrm{kg}$ & 0.01 & $\mathrm{Rh}$ & $\mathrm{mg} / \mathrm{kg}$ & 0.01 \\
\hline $\mathrm{Cu}$ & $\mathrm{mg} / \mathrm{kg}$ & 0.01 & $\mathrm{Ru}$ & $\mu \mathrm{g} / \mathrm{kg}$ & 0.1 \\
\hline Dy & $\mathrm{mg} / \mathrm{kg}$ & 0.01 & $\mathrm{Sb}$ & $\mathrm{mg} / \mathrm{kg}$ & 0.01 \\
\hline $\mathrm{Er}$ & $\mathrm{mg} / \mathrm{kg}$ & 0.01 & Sc & $\mathrm{mg} / \mathrm{kg}$ & 0.1 \\
\hline Eu & $\mathrm{mg} / \mathrm{kg}$ & 0.01 & Sm & $\mathrm{mg} / \mathrm{kg}$ & 0.1 \\
\hline $\mathrm{Fe}$ & $\%$ & 0.01 & Sn & $\mathrm{mg} / \mathrm{kg}$ & 0.01 \\
\hline $\mathrm{Ga}$ & $\mathrm{mg} / \mathrm{kg}$ & 0.01 & $\mathrm{Sr}$ & $\mathrm{mg} / \mathrm{kg}$ & 0.01 \\
\hline Gd & $\mathrm{mg} / \mathrm{kg}$ & 0.01 & $\mathrm{Ta}$ & $\mathrm{mg} / \mathrm{kg}$ & 0.1 \\
\hline $\mathrm{Ge}$ & $\mathrm{mg} / \mathrm{kg}$ & 0.01 & $\mathrm{~Tb}$ & $\mathrm{mg} / \mathrm{kg}$ & 0.01 \\
\hline $\mathrm{Hf}$ & $\mathrm{mg} / \mathrm{kg}$ & 0.01 & $\mathrm{Te}$ & $\mu \mathrm{g} / \mathrm{kg}$ & 0.01 \\
\hline $\mathrm{Hg}$ & $\mathrm{mg} / \mathrm{kg}$ & 0.01 & Th & $\mu \mathrm{g} / \mathrm{kg}$ & 0.1 \\
\hline Ho & $\mathrm{mg} / \mathrm{kg}$ & 0.01 & $\mathrm{Ti}$ & $\%$ & 0.1 \\
\hline 1 & $\mathrm{mg} / \mathrm{kg}$ & 0.01 & $\mathrm{TI}$ & $\mathrm{mg} / \mathrm{kg}$ & 0.1 \\
\hline In & $\mu \mathrm{g} / \mathrm{kg}$ & 0.01 & $\mathrm{Tm}$ & $\mathrm{mg} / \mathrm{kg}$ & 0.01 \\
\hline Ir & $\mu \mathrm{g} / \mathrm{kg}$ & 0.01 & V & $\mathrm{mg} / \mathrm{kg}$ & 0.01 \\
\hline K & $\%$ & 0.01 & W & $\mathrm{mg} / \mathrm{kg}$ & 0.01 \\
\hline La & $\mathrm{mg} / \mathrm{kg}$ & 0.01 & $Y$ & $\mathrm{mg} / \mathrm{kg}$ & 0.01 \\
\hline $\mathrm{Li}$ & $\mathrm{mg} / \mathrm{kg}$ & 0.01 & $\mathrm{Yb}$ & $\mathrm{mg} / \mathrm{kg}$ & 0.01 \\
\hline Lu & $\mathrm{mg} / \mathrm{kg}$ & 0.01 & $\mathrm{Zn}$ & $\mathrm{mg} / \mathrm{kg}$ & 0.01 \\
\hline $\mathrm{Mg}$ & $\%$ & 0.1 & $\mathrm{Zr}$ & $\mathrm{mg} / \mathrm{kg}$ & 0.01 \\
\hline
\end{tabular}

LOD-limit of detection. 
samples were analyzed at a combined frequency of $20 \%$ of the samples. The recovery for all of the analyzed elements ranges from $76.8 \%$ for $\mathrm{Tl}$ to $119 \%$ for $\mathrm{Sb}$ (for ICP-MS measurements) and from $87.5 \%$ for $\mathrm{Na}$ to $112 \%$ for $\mathrm{P}$ (for ICP-AES measurements).

\section{Data processing}

The data for the contents of the investigated elements were statistically processed using statistical software (Stat Soft, 11.0, Dell Software, Aliso Viejo, CA, USA) and conducting parametric and non-parametric analysis. Basic descriptive statistical analysis was conducted on the values of the contents of the elements in all types of samples. At the same time, normalization tests were conducted and based on the obtained results and the visual check of distribution histograms, the distribution of the data for the independent variables (elements' contents) was determined. For data normalization, the method of Box-Cox transformation was also applied. ${ }^{[27]}$ The F-test was applied for processing of certain data for the needs of comparative analysis. Factor analysis (FA) and cluster analysis (CA) were used as methods for multivariate analysis. ${ }^{[28]}$ In the preparation of the distribution maps the Kriging method with linear variogram interpolation was applied. Percentile values of the distribution of the interpolated values were considered as area limits. ${ }^{[29]}$

\section{Results and discussion}

\section{Lithogenic and anthropogenic distribution in sediment and fluvisol}

The complete overview of the elements' contents, i.e. the values of the medians in the sediments and alluvial soils, is presented

Table 3. Descriptive statistics for the values of elements' contents in samples of alluvial soil ( $\mathrm{N}=32$, for the whole basin).

\begin{tabular}{|c|c|c|c|c|c|c|c|c|c|c|c|}
\hline \multirow[b]{2}{*}{ Element } & \multirow[b]{2}{*}{ Unit } & \multicolumn{5}{|c|}{ Sediment } & \multicolumn{5}{|c|}{ Fluvisol } \\
\hline & & Min & $P_{25}$ & Median & $P_{75}$ & Max & Min & $P_{25}$ & Median & $P_{75}$ & Max \\
\hline $\mathrm{Al}$ & $\%$ & 3.3 & 5.8 & 6.1 & 6.4 & 7.3 & 4.8 & 5.6 & 6.1 & 6.5 & 6.9 \\
\hline $\mathrm{Ca}$ & $\%$ & 0.67 & 1.7 & 1.9 & 2.6 & 5.4 & 0.97 & 1.4 & 1.8 & 2.2 & 4.0 \\
\hline $\mathrm{Fe}$ & $\%$ & 1.3 & 5.0 & 6.2 & 7.4 & 39 & 0.92 & 2.5 & 3.3 & 4.6 & 24 \\
\hline $\mathrm{K}$ & $\%$ & 0.028 & 0.082 & 0.29 & 0.49 & 1.4 & 0.018 & 0.069 & 0.32 & 0.89 & 1.4 \\
\hline $\mathrm{Mg}$ & $\%$ & 0.54 & 0.87 & 1.0 & 1.4 & 4.7 & 0.25 & 0.44 & 0.63 & 0.85 & 5.0 \\
\hline $\mathrm{Na}$ & $\%$ & 0.69 & 1.6 & 3.2 & 5.3 & 18 & 0.62 & 1.2 & 1.7 & 4.0 & 13 \\
\hline$P$ & $\%$ & 0.056 & 0.083 & 0.10 & 0.11 & 0.35 & 0.032 & 0.057 & 0.073 & 0.086 & 0.33 \\
\hline $\mathrm{Ti}$ & $\%$ & 0.071 & 0.26 & 0.50 & 0.62 & 0.93 & 0.053 & 0.13 & 0.24 & 0.33 & 0.65 \\
\hline $\mathrm{Ag}$ & $\mathrm{mg} / \mathrm{kg}$ & 0.34 & 0.82 & 1.0 & 1.5 & 4.6 & 0.27 & 0.35 & 0.72 & 1.1 & 2.9 \\
\hline As & $\mathrm{mg} / \mathrm{kg}$ & 7.9 & 14 & 18 & 28 & 140 & 5.3 & 7.9 & 13 & 21 & 130 \\
\hline$B$ & $\mathrm{mg} / \mathrm{kg}$ & 0.071 & 20 & 33 & 42 & 86 & 0.005 & 0.005 & 0.41 & 1.0 & 8.9 \\
\hline $\mathrm{Ba}$ & $\mathrm{mg} / \mathrm{kg}$ & 240 & 470 & 630 & 780 & 2100 & 240 & 340 & 470 & 580 & 2300 \\
\hline $\mathrm{Be}$ & $\mathrm{mg} / \mathrm{kg}$ & 0.65 & 1.6 & 2.1 & 3.0 & 5.3 & 0.58 & 0.95 & 1.6 & 1.8 & 4.7 \\
\hline $\mathrm{Bi}$ & $\mathrm{mg} / \mathrm{kg}$ & 0.005 & 0.15 & 0.25 & 0.39 & 19 & 0.005 & 0.032 & 0.065 & 0.22 & 2.5 \\
\hline $\mathrm{Br}$ & $\mathrm{mg} / \mathrm{kg}$ & 0.005 & 0.005 & 1.1 & 2.9 & 6.6 & 0.005 & 0.005 & 1.1 & 1.7 & 5.4 \\
\hline $\mathrm{Cd}$ & $\mathrm{mg} / \mathrm{kg}$ & 0.062 & 0.24 & 0.35 & 1.1 & 22 & 0.005 & 0.051 & 0.11 & 0.44 & 3.5 \\
\hline Co & $\mathrm{mg} / \mathrm{kg}$ & 5.6 & 11 & 15 & 23 & 52 & 4.2 & 5.9 & 7.6 & 12 & 40 \\
\hline $\mathrm{Cr}$ & $\mathrm{mg} / \mathrm{kg}$ & 24 & 57 & 74 & 100 & 180 & 17 & 25 & 41 & 52 & 170 \\
\hline Cs & $\mathrm{mg} / \mathrm{kg}$ & 1.4 & 2.3 & 3.2 & 4.6 & 20 & 0.26 & 1.2 & 1.9 & 2.6 & 18 \\
\hline $\mathrm{Cu}$ & $\mathrm{mg} / \mathrm{kg}$ & 20 & 36 & 51 & 99 & 430 & 7.6 & 15 & 28 & 54 & 180 \\
\hline $\mathrm{Ga}$ & $\mathrm{mg} / \mathrm{kg}$ & 6.6 & 16 & 25 & 32 & 72 & 4.9 & 7.5 & 12 & 16 & 50 \\
\hline $\mathrm{Ge}$ & $\mathrm{mg} / \mathrm{kg}$ & 0.005 & 1.1 & 1.4 & 1.7 & 3.8 & 0.005 & 0.24 & 0.51 & 0.73 & 3.1 \\
\hline $\mathrm{Hf}$ & $\mathrm{mg} / \mathrm{kg}$ & 0.31 & 0.77 & 1.0 & 1.6 & 3.9 & 0.22 & 0.34 & 0.63 & 0.83 & 3.0 \\
\hline I & $\mathrm{mg} / \mathrm{kg}$ & 0.005 & 0.089 & 0.23 & 0.47 & 0.98 & 0.005 & 0.005 & 0.032 & 0.061 & 0.13 \\
\hline In & $\mu \mathrm{g} / \mathrm{kg}$ & 5.0 & 46 & 93 & 120 & 790 & 5.0 & 13 & 24 & 81 & 560 \\
\hline $\mathrm{Li}$ & $\mathrm{mg} / \mathrm{kg}$ & 10 & 17 & 32 & 47 & 82 & 3.7 & 12 & 15 & 22 & 76 \\
\hline $\mathrm{Mn}$ & $\mathrm{mg} / \mathrm{kg}$ & 340 & 1000 & 1600 & 3100 & 9900 & 340 & 440 & 650 & 1200 & 12000 \\
\hline Mo & $\mathrm{mg} / \mathrm{kg}$ & 0.042 & 0.61 & 0.95 & 1.5 & 5.2 & 0.005 & 0.20 & 0.41 & 0.93 & 4.5 \\
\hline $\mathrm{Nb}$ & $\mathrm{mg} / \mathrm{kg}$ & 4.1 & 8.5 & 13 & 18 & 31 & 2.8 & 4.4 & 6.5 & 7.8 & 19 \\
\hline $\mathrm{Ni}$ & $\mathrm{mg} / \mathrm{kg}$ & 12 & 20 & 32 & 41 & 93 & 3.4 & 9.3 & 14 & 20 & 54 \\
\hline $\mathrm{Pb}$ & $\mathrm{mg} / \mathrm{kg}$ & 18 & 25 & 32 & 68 & 11000 & 6.7 & 13 & 26 & 59 & 34000 \\
\hline $\mathrm{Pd}$ & $\mathrm{mg} / \mathrm{kg}$ & 0.050 & 0.24 & 0.77 & 1.9 & 6.4 & 0.050 & 0.12 & 0.20 & 0.48 & 4.5 \\
\hline $\mathrm{Pt}$ & $\mu \mathrm{g} / \mathrm{kg}$ & 95 & 180 & 230 & 270 & 600 & 5.0 & 9.4 & 20 & 92 & 210 \\
\hline $\mathrm{Rb}$ & $\mathrm{mg} / \mathrm{kg}$ & 30 & 56 & 82 & 120 & 230 & 16 & 43 & 52 & 64 & 290 \\
\hline $\mathrm{Sb}$ & $\mathrm{mg} / \mathrm{kg}$ & 0.21 & 0.32 & 0.46 & 1.0 & 4.6 & 0.13 & 0.21 & 0.43 & 0.72 & 6.4 \\
\hline Sc & $\mathrm{mg} / \mathrm{kg}$ & 1.6 & 5.4 & 13 & 17 & 23 & 0.90 & 2.9 & 7.8 & 13 & 21 \\
\hline Sn & $\mathrm{mg} / \mathrm{kg}$ & 0.005 & 3.5 & 4.7 & 6.0 & 15 & 0.41 & 1.2 & 1.7 & 2.1 & 5.6 \\
\hline $\mathrm{Sr}$ & $\mathrm{mg} / \mathrm{kg}$ & 98 & 170 & 230 & 310 & 1100 & 62 & 84 & 120 & 190 & 1200 \\
\hline $\mathrm{Ta}$ & $\mathrm{mg} / \mathrm{kg}$ & 0.36 & 0.80 & 1.2 & 1.6 & 2.9 & 0.25 & 0.45 & 0.61 & 0.80 & 2.3 \\
\hline $\mathrm{Te}$ & $\mu \mathrm{g} / \mathrm{kg}$ & 5.0 & 15 & 32 & 60 & 2000 & 5.0 & 9.3 & 21 & 49 & 420 \\
\hline TI & $\mathrm{mg} / \mathrm{kg}$ & 0.13 & 0.35 & 0.48 & 0.70 & 2.7 & 0.050 & 0.21 & 0.30 & 0.43 & 4.5 \\
\hline V & $\mathrm{mg} / \mathrm{kg}$ & 53 & 97 & 150 & 210 & 910 & 37 & 51 & 74 & 100 & 500 \\
\hline W & $\mathrm{mg} / \mathrm{kg}$ & 0.73 & 1.2 & 1.5 & 2.2 & 10 & 0.20 & 0.68 & 0.90 & 1.3 & 3.5 \\
\hline$Y$ & $\mathrm{mg} / \mathrm{kg}$ & 8.5 & 18 & 29 & 41 & 80 & 5.3 & 8.9 & 12 & 18 & 48 \\
\hline $\mathrm{Zn}$ & $\mathrm{mg} / \mathrm{kg}$ & 25 & 86 & 120 & 300 & 3400 & 10 & 30 & 55 & 140 & 930 \\
\hline $\mathrm{Zr}$ & $\mathrm{mg} / \mathrm{kg}$ & 8.9 & 24 & 34 & 49 & 130 & 6.3 & 10 & 19 & 26 & 110 \\
\hline
\end{tabular}

$P_{25}-25$ percentile; $P_{75}-75$ percentile. 
in Table 3. The contents of the macro, micro and elements in traces in the first place depend on the geochemical processes and hydrographic conditions, as well as on the mechanical structure of the alluvial soils, as stated by Du Laing et al. ${ }^{[10]}$ Of all 69 analyzed elements, the contents of only five elements were not determined as their presence was below the detection limit ( $\mathrm{Au}, \mathrm{Os}, \mathrm{Re}, \mathrm{Ru}$ and $\mathrm{Th}$ ). The basic, matrix elements (Al,
$\mathrm{Ca}, \mathrm{Mg}, \mathrm{K}, \mathrm{Fe}, \mathrm{Na}, \mathrm{P}$ and $\mathrm{Ti}$ ) show significant stability with insignificant variations along the whole course of the river Bregalnica. The concentration of the major elements $\mathrm{Na}, \mathrm{K}, \mathrm{Ca}$, and $\mathrm{Mg}$ was usually associated with the increase of the soil salinity. ${ }^{[8]}$ The addition of Ca-salts usually results in a higher release of exchangeable metals in the soil solution compared to the addition of Na-salts, which are less competitive for

Table 4. T-test and F-test for the contents of the elements in the sediments of the different zones in the course of the Bregalnica river.

\begin{tabular}{|c|c|c|c|c|c|c|c|c|c|c|c|c|}
\hline \multirow[b]{2}{*}{ Element } & \multicolumn{4}{|c|}{ Zone 1 vs. Zone 2} & \multicolumn{4}{|c|}{ Zone 2 vs. Zone 3} & \multicolumn{4}{|c|}{ Zone 3 vs. Zone 4} \\
\hline & $\mathrm{t}$ & $P$ & $\mathrm{~F}$ & $P($ var $)$ & $\mathrm{T}$ & $P$ & $\mathrm{~F}$ & $P($ var $)$ & $\mathrm{t}$ & $P$ & $\mathrm{~F}$ & $P($ var $)$ \\
\hline $\mathrm{Al}$ & 0.25 & $0.80^{* *}$ & 1.57 & 0.22 & -1.35 & $0.19^{*}$ & 1.25 & 0.75 & -0.25 & $0.80^{* *}$ & 1.85 & 0.35 \\
\hline $\mathrm{Ca}$ & 1.22 & $0.23^{*}$ & 1.82 & 0.10 & 0.83 & $0.41^{*}$ & 1.05 & 1.00 & -0.71 & $0.49^{*}$ & 2.25 & 0.22 \\
\hline$P$ & 3.89 & 0.001 & 1.81 & 0.10 & 0.14 & $0.89^{* *}$ & 2.94 & 0.07 & 0.14 & $0.89^{* *}$ & 1.82 & 0.36 \\
\hline $\mathrm{Ag}$ & 2.97 & 0.001 & 1.54 & 0.24 & -0.11 & $0.91^{* *}$ & 4.30 & 0.02 & 0.24 & $0.81^{* *}$ & 1.70 & 0.42 \\
\hline As & 2.33 & 0.02 & 1.90 & 0.08 & 3.12 & 0.001 & 1.52 & 0.49 & -1.63 & $0.12^{*}$ & 1.06 & 0.93 \\
\hline B & 10.6 & 0.001 & 1.74 & 0.13 & 0.30 & $0.76^{* *}$ & 1.39 & 0.60 & 0.07 & $0.94^{* *}$ & 1.58 & 0.48 \\
\hline $\mathrm{Ba}$ & 2.15 & 0.04 & 1.09 & 0.82 & 0.70 & $0.49^{*}$ & 1.86 & 0.30 & -0.25 & $0.81^{* *}$ & 1.24 & 0.74 \\
\hline $\mathrm{Be}$ & 2.83 & 0.01 & 1.10 & 0.78 & 0.40 & $0.69^{* *}$ & 4.11 & 0.02 & -0.83 & $0.42^{*}$ & 1.26 & 0.72 \\
\hline $\mathrm{Bi}$ & 2.95 & 0.001 & 1.01 & 0.98 & -0.30 & $0.76^{* *}$ & 2.65 & 0.11 & 0.25 & $0.80^{* *}$ & 1.83 & 0.35 \\
\hline $\mathrm{Br}$ & 0.12 & $0.90^{* *}$ & 1.47 & 0.29 & -0.70 & $0.49^{*}$ & 2.32 & 0.16 & -0.42 & $0.68^{* *}$ & 1.99 & 0.29 \\
\hline $\mathrm{Cd}$ & 3.14 & 0.001 & 1.59 & 0.20 & -0.71 & $0.48^{*}$ & 3.13 & 0.06 & 0.12 & $0.91^{* *}$ & 2.28 & 0.21 \\
\hline $\mathrm{Ce}$ & 5.13 & 0.001 & 1.31 & 0.46 & 1.55 & $0.13^{*}$ & 2.96 & 0.07 & -1.07 & $0.30^{*}$ & 1.59 & 0.47 \\
\hline Co & 4.64 & 0.001 & 1.60 & 0.20 & 2.03 & 0.05 & 2.19 & 0.19 & -1.44 & $0.17^{*}$ & 1.61 & 0.46 \\
\hline $\mathrm{Cr}$ & 5.04 & 0.001 & 1.38 & 0.38 & 1.25 & $0.22^{*}$ & 1.40 & 0.59 & -1.12 & $0.28^{*}$ & 1.33 & 0.66 \\
\hline Cs & 3.55 & 0.001 & 1.97 & 0.06 & 0.21 & $0.84^{* *}$ & 9.15 & 0.00 & -0.85 & $0.40^{*}$ & 2.08 & 0.26 \\
\hline $\mathrm{Cu}$ & 3.99 & 0.001 & 2.16 & 0.04 & 2.46 & 0.02 & 4.45 & 0.02 & -1.09 & $0.29^{*}$ & 1.73 & 0.40 \\
\hline Dy & 5.03 & 0.001 & 1.17 & 0.67 & 1.36 & $0.18^{*}$ & 2.60 & 0.11 & -0.71 & $0.49^{*}$ & 1.38 & 0.62 \\
\hline $\mathrm{Er}$ & 4.84 & 0.001 & 1.13 & 0.74 & 1.18 & $0.25^{*}$ & 2.39 & 0.15 & -0.51 & $0.62^{* *}$ & 1.15 & 0.83 \\
\hline $\mathrm{Eu}$ & 5.15 & 0.001 & 1.23 & 0.56 & 2.38 & 0.02 & 2.33 & 0.16 & -1.26 & $0.22^{*}$ & 1.41 & 0.60 \\
\hline $\mathrm{Fe}$ & 3.21 & 0.001 & 1.16 & 0.69 & 2.39 & 0.02 & 11.95 & 0.00 & -1.24 & $0.23^{*}$ & 4.62 & 0.02 \\
\hline $\mathrm{Ga}$ & 4.18 & 0.001 & 1.00 & 1.00 & 1.39 & $0.17^{*}$ & 2.36 & 0.15 & -0.79 & $0.44^{*}$ & 1.66 & 0.44 \\
\hline $\mathrm{Gd}$ & 5.34 & 0.001 & 1.24 & 0.56 & 1.50 & $0.14^{*}$ & 2.82 & 0.09 & -0.84 & $0.41^{*}$ & 1.60 & 0.47 \\
\hline $\mathrm{Ge}$ & 4.63 & 0.001 & 1.07 & 0.85 & -0.05 & $0.96^{* *}$ & 2.47 & 0.13 & -0.12 & $0.91^{* *}$ & 1.82 & 0.36 \\
\hline $\mathrm{Hf}$ & 3.79 & 0.001 & 1.47 & 0.29 & 0.62 & $0.54^{*}$ & 5.70 & 0.01 & -0.07 & $0.94^{* *}$ & 1.36 & 0.64 \\
\hline $\mathrm{Ho}$ & 4.95 & 0.001 & 1.18 & 0.64 & 1.24 & $0.22^{*}$ & 2.84 & 0.08 & -0.72 & $0.48^{*}$ & 1.35 & 0.65 \\
\hline 1 & 5.26 & 0.001 & 2.08 & 0.05 & -1.45 & $0.15^{*}$ & 2.09 & 0.22 & 0.22 & $0.83^{* *}$ & 1.72 & 0.41 \\
\hline In & 2.46 & 0.02 & 1.01 & 0.99 & -0.36 & $0.72^{* *}$ & 2.90 & 0.08 & 0.43 & $0.67^{* *}$ & 1.82 & 0.36 \\
\hline $\mathrm{K}$ & -0.90 & $0.37^{*}$ & 1.74 & 0.13 & -1.85 & $0.07^{*}$ & 1.63 & 0.42 & 0.50 & $0.63^{* *}$ & 1.69 & 0.42 \\
\hline $\mathrm{La}$ & 5.44 & 0.001 & 1.42 & 0.33 & 1.50 & $0.14^{*}$ & 3.14 & 0.06 & -0.93 & $0.37^{*}$ & 1.66 & 0.44 \\
\hline $\mathrm{Li}$ & 4.27 & 0.001 & 1.38 & 0.37 & 0.20 & $0.84^{* *}$ & 11.57 & 0.00 & -1.33 & $0.20^{*}$ & 2.79 & 0.12 \\
\hline $\mathrm{Lu}$ & 4.86 & 0.001 & 1.09 & 0.80 & 1.20 & $0.24^{*}$ & 2.63 & 0.11 & -0.54 & $0.59^{*}$ & 1.28 & 0.71 \\
\hline $\mathrm{Mg}$ & 4.42 & 0.001 & 2.11 & 0.04 & 2.14 & 0.04 & 1.82 & 0.32 & -1.01 & $0.32^{*}$ & 1.09 & 0.89 \\
\hline $\mathrm{Mn}$ & 3.43 & 0.001 & 1.22 & 0.58 & 1.45 & $0.15^{*}$ & 1.67 & 0.39 & -0.53 & $0.60^{\text {** }}$ & 1.29 & 0.69 \\
\hline Mo & 2.54 & 0.01 & 1.08 & 0.83 & 1.21 & $0.23^{*}$ & 2.03 & 0.24 & -0.27 & $0.79^{* *}$ & 2.52 & 0.16 \\
\hline $\mathrm{Na}$ & 2.05 & 0.04 & 1.17 & 0.66 & 3.19 & 0.001 & 3.80 & 0.03 & -0.72 & $0.48^{*}$ & 1.03 & 0.97 \\
\hline $\mathrm{Nb}$ & 5.11 & 0.001 & 1.03 & 0.93 & 1.34 & $0.19^{*}$ & 2.25 & 0.18 & -0.23 & $0.82^{* *}$ & 1.46 & 0.56 \\
\hline $\mathrm{Nd}$ & 5.28 & 0.001 & 1.30 & 0.48 & 1.70 & $0.10^{*}$ & 3.13 & 0.06 & -0.97 & $0.34^{*}$ & 1.55 & 0.50 \\
\hline $\mathrm{Ni}$ & 5.21 & 0.001 & 2.14 & 0.04 & 0.82 & $0.42^{*}$ & 1.15 & 0.86 & -1.07 & $0.30^{*}$ & 1.41 & 0.60 \\
\hline $\mathrm{Pb}$ & 1.77 & $0.08^{*}$ & 2.18 & 0.03 & -0.51 & $0.61^{* *}$ & 1.71 & 0.37 & 0.18 & $0.86^{* *}$ & 1.07 & 0.92 \\
\hline $\mathrm{Pd}$ & 2.65 & 0.01 & 1.69 & 0.15 & -2.07 & $0.04^{*}$ & 1.66 & 0.40 & 0.66 & $0.51^{*}$ & 1.69 & 0.42 \\
\hline $\operatorname{Pr}$ & 5.38 & 0.001 & 1.31 & 0.46 & 1.65 & $0.11^{*}$ & 3.19 & 0.06 & -1.06 & $0.30^{*}$ & 1.62 & 0.46 \\
\hline $\mathrm{Pt}$ & 9.01 & 0.001 & 8.42 & 0.00 & 2.34 & $0.02^{*}$ & 39.93 & 0.00 & 1.61 & $0.12^{*}$ & 6.21 & 0.01 \\
\hline $\mathrm{Rb}$ & 3.34 & 0.001 & 1.42 & 0.34 & 1.17 & $0.25^{*}$ & 5.82 & 0.01 & -1.07 & $0.30^{*}$ & 2.37 & 0.19 \\
\hline $\mathrm{Sb}$ & 1.57 & $0.12^{*}$ & 1.38 & 0.38 & 0.24 & $0.81^{* *}$ & 4.07 & 0.02 & -0.59 & $0.56^{*}$ & 1.78 & 0.38 \\
\hline Sc & 2.42 & 0.02 & 1.00 & 1.00 & -1.02 & $0.31^{*}$ & 1.03 & 0.88 & -0.31 & $0.76^{* *}$ & 1.32 & 0.67 \\
\hline Sm & 5.36 & 0.001 & 1.24 & 0.55 & 1.74 & $0.09^{*}$ & 2.74 & 0.09 & -0.98 & $0.34^{*}$ & 1.40 & 0.61 \\
\hline Sn & 4.78 & 0.001 & 4.80 & 0.00 & 1.52 & $0.14^{*}$ & 2.80 & 0.09 & -1.33 & $0.20^{*}$ & 2.00 & 0.29 \\
\hline $\mathrm{Sr}$ & 4.27 & 0.001 & 1.98 & 0.06 & 1.16 & $0.25^{*}$ & 2.16 & 0.20 & -1.12 & $0.28^{*}$ & 1.41 & 0.60 \\
\hline $\mathrm{Ta}$ & 4.38 & 0.001 & 1.49 & 0.27 & 1.78 & $0.08^{*}$ & 3.02 & 0.07 & -0.25 & $0.81^{* *}$ & 1.10 & 0.88 \\
\hline $\mathrm{Tb}$ & 5.28 & 0.001 & 1.23 & 0.57 & 1.38 & $0.18^{*}$ & 2.95 & 0.07 & -0.81 & $0.43^{*}$ & 1.54 & 0.51 \\
\hline $\mathrm{Te}$ & 1.28 & $0.20^{*}$ & 1.13 & 0.74 & 0.16 & $0.87^{* *}$ & 2.66 & 0.10 & 0.35 & $0.73^{* *}$ & 1.48 & 0.55 \\
\hline $\mathrm{Ti}$ & 4.10 & 0.001 & 1.57 & 0.22 & -0.31 & $0.76^{* *}$ & 1.11 & 0.91 & -0.18 & $0.86^{* *}$ & 1.15 & 0.83 \\
\hline $\mathrm{Tl}$ & 2.61 & 0.01 & 1.91 & 0.08 & -0.08 & $0.94^{* *}$ & 5.14 & 0.01 & -0.40 & $0.69^{* *}$ & 1.52 & 0.52 \\
\hline $\mathrm{Tm}$ & 4.55 & 0.001 & 1.03 & 0.93 & 1.15 & $0.26^{*}$ & 2.73 & 0.10 & -0.52 & $0.61^{* *}$ & 1.29 & 0.70 \\
\hline V & 4.50 & 0.001 & 1.43 & 0.32 & 1.84 & $0.07^{*}$ & 2.11 & 0.21 & -1.03 & $0.32^{*}$ & 1.17 & 0.81 \\
\hline W & 4.24 & 0.001 & 1.69 & 0.15 & 0.78 & $0.44^{*}$ & 7.95 & 0.00 & -0.34 & $0.74^{* *}$ & 1.78 & 0.38 \\
\hline$Y$ & 4.88 & 0.001 & 1.12 & 0.75 & 1.28 & $0.21^{*}$ & 3.11 & 0.06 & -0.69 & $0.50^{*}$ & 1.64 & 0.45 \\
\hline $\mathrm{Yb}$ & 4.65 & 0.001 & 1.04 & 0.92 & 1.13 & $0.26^{*}$ & 2.41 & 0.14 & -0.45 & $0.66^{* *}$ & 1.22 & 0.76 \\
\hline $\mathrm{Zn}$ & 3.17 & 0.001 & 1.76 & 0.12 & -0.15 & $0.88^{* *}$ & 5.91 & 0.01 & -0.19 & $0.85^{* *}$ & 1.40 & 0.61 \\
\hline $\mathrm{Zr}$ & 3.74 & 0.001 & 1.50 & 0.27 & 0.53 & $0.60^{* *}$ & 6.79 & 0.00 & 0.09 & $0.93^{* *}$ & 1.89 & 0.33 \\
\hline
\end{tabular}

T- t value (T-test), F- F value, ${ }^{*}$ - Significant difference at the 0.10 level, $^{* *}$ - Significant difference at the 0.05 level. 
sorption. ${ }^{[12]}$ The sediments are characterized with median value of Ca contents of $1.9 \%$ and $1.8 \%$ in fluvisol vs. $1.6 \%$ and $1.7 \%$ for $\mathrm{Na}$ contents respectively (Table 3 ). Aluminum is present in the highest concentration in the sediment $(6.6 \%)$ of the fourth zone of the Bregalnica river course, with almost insignificant variations to $5.5 \%$ in the alluvium of the river Lakavica. Of all matrix geogenic elements, only iron shows a trend of variability of its median values in the sediment and the alluvial soils in the whole basin of the river Bregalnica. Iron is usually the most predominant element that generates sulphide precipitates. As Du Laing et al. ${ }^{[10]}$ report, it is released from reducible minerals such as oxides/oxihydroxides within the sedimentary layers, where sulphate reduction occurs, which results in ferrous Fe. A major difference in the Fe contents between the sediment and the fluvisol (6.5 and 6.1\%, respectively) was not detected in the upper course of the river, while in the second, third and fourth zone a trend of lower Fe content in the alluvium as opposed to the sediment was perceived (Table 3). The maximum iron content (29\%) was detected in the sediment of the Zletovska River. Generally, Fe occurs in soluble forms in acid environment. When a neutral $\mathrm{pH}$ is approached, Fe can only exist in insoluble form with low redox potentials or as a soluble organic complex in oxic soils. ${ }^{[10]}$ These authors also reported that the reduction of $\mathrm{Fe}^{3+}$ does not start before the complete depletion of all $\mathrm{Mn}^{4+}$. The total manganese contents in the river Bregalnica alluvial soil ranges from 0.034 to $1.2 \%$.

Copper was found in higher contents in the sediments of the investigated area, in certain zones along the Bregalnica river course. In accordance with the Dutch standards (http:// www.contaminatedland.co.uk/std-guid/dutch-l.htm), the optimum contents of $\mathrm{Cu}$ range up to $36 \mathrm{mg} / \mathrm{kg}$. As presented in Table 3, along the whole course of the Bregalnica River (Zones 1-4), the Kamenička River and the Zletovska River, the copper contents in the sediment exceed the optimal value in accordance with the previously mentioned standards, while in the sediment of the Kamenička River the $\mathrm{Cu}$ contents exceed the action value $(190 \mathrm{mg} / \mathrm{kg})$. The copper content in the sediment of the Kamenička River amounts to $290 \mathrm{mg} / \mathrm{kg}$.

Regarding cadmium contents, it is found in the sediment in maximum content of $22 \mathrm{mg} / \mathrm{kg}$, while in the fluvisol the content of this element is not higher than $3.5 \mathrm{mg} / \mathrm{kg}$ (Table 3). Taking into consideration the Dutch standards for Cd contents in sediment (optimum value $0.8 \mathrm{mg} \mathrm{kg}^{-1}$ and action value $12 \mathrm{mg} / \mathrm{kg}$ ), there is a significant increase in Cd contents in the sediment in the third zone along the course of the river Bregalnica. On the other hand, in the sediment of the Kamenička River, a median value of $15 \mathrm{mg} / \mathrm{kg}$ (Table 3 ) was obtained.

The cobalt contents do not show any significant variations in the sediment of the Bregalnica basin, except for the fact that in zone 2 along the course of the river Bregalnica, a value at the upper limit of the optimum value in accordance with the Dutch standards $(25 \mathrm{mg} / \mathrm{kg})$ was obtained. Lead is also found in contents that point to environment pollution. Maximum $\mathrm{Pb}$ contents were detected in the sediment of the Kamenička River $(1.1 \%)$, and in the alluvial soils, as high as up to $3.4 \%$. These values point to the characteristic negative effect of the $\mathrm{Pb}-\mathrm{Zn}$ ore processing in the ore and flotation plants of the Sasa mine. The optimum values for the $\mathrm{Pb}$ contents in sediment according to the Dutch standards amounts to $85 \mathrm{mg} / \mathrm{kg}$, while the action value is $530 \mathrm{mg} / \mathrm{kg}$. The $\mathrm{Pb}$ contents in the sediment in the three zones from the river source to the Zletovska river mouth do not exceed the relevant Dutch optimum values. Nevertheless, for the lead content in the sediment of the Kamenička River, a median of $7100 \mathrm{mg} / \mathrm{kg}$ was obtained, while for the Zletovska River a median of $670 \mathrm{mg} / \mathrm{kg}$ was obtained (Table 3). The sediment is a natural archive of a great number of chemical parameters, and especially of various chemical elements, with particular accent on the potentially toxic metals. Therefore, it can be said that the area around the Sasa mine, i.e. the Kamenička River is exposed to higher concentration of $\mathrm{Pb}$, which exceed the relevant action values of $\mathrm{Pb}$ in relation to the Dutch standards $(530 \mathrm{mg} / \mathrm{kg})$. Similarly to the lead, the zinc contents in the sediment also show a comparable trend of deposition, owing to the intensive introduction of these elements related to the activities in the ore plants and the ore flotation processes in the Sasa and Zletovo mines. The zinc contents in the sediment exceed the optimum values in accordance with the Dutch standards $(140 \mathrm{mg} / \mathrm{kg})$ in zones 3 and 4 along the river Bregalnica, as well as in the sediment of the Kamenička $(2200 \mathrm{mg} / \mathrm{kg})$ and Zletovska River $(1000 \mathrm{mg} / \mathrm{kg})$. The zinc content has a maximum value of

Table 5. Matrix of factor loadings-factor analysis (FA) of the elements' contents in samples of sediment and fluvisol.

\begin{tabular}{|c|c|c|c|c|}
\hline Element & $\mathrm{F} 1$ & $\mathrm{~F} 2$ & F3 & Communality \\
\hline$Y$ & 0.95 & 0.19 & 0.10 & 94.2 \\
\hline Eu-Lu & 0.94 & 0.20 & 0.12 & 92.8 \\
\hline $\mathrm{Cr}$ & 0.89 & 0.13 & 0.18 & 84.7 \\
\hline V & 0.89 & 0.37 & 0.12 & 94.4 \\
\hline La-Gd & 0.87 & 0.38 & 0.09 & 91.6 \\
\hline $\mathrm{Nb}$ & 0.87 & 0.32 & 0.19 & 89.2 \\
\hline Co & 0.87 & 0.38 & 0.10 & 90.6 \\
\hline $\mathrm{Hf}$ & 0.85 & 0.20 & -0.05 & 77.1 \\
\hline $\mathrm{Zr}$ & 0.85 & 0.23 & 0.01 & 77.5 \\
\hline $\mathrm{Ga}$ & 0.85 & 0.42 & 0.19 & 93.6 \\
\hline $\mathrm{Mg}$ & 0.84 & 0.29 & -0.16 & 82.4 \\
\hline $\mathrm{Fe}$ & 0.81 & 0.37 & -0.32 & 89.6 \\
\hline $\mathrm{Sr}$ & 0.78 & 0.33 & 0.11 & 73.3 \\
\hline $\mathrm{Ta}$ & 0.78 & 0.21 & 0.17 & 67.9 \\
\hline Sn & 0.77 & 0.39 & -0.08 & 74.8 \\
\hline $\mathrm{Li}$ & 0.76 & 0.42 & 0.18 & 78.3 \\
\hline $\mathrm{Na}$ & 0.74 & 0.23 & -0.30 & 69.9 \\
\hline $\mathrm{Rb}$ & 0.73 & 0.50 & 0.20 & 82.2 \\
\hline $\mathrm{Ni}$ & 0.69 & 0.21 & 0.19 & 56.3 \\
\hline $\mathrm{Ge}$ & 0.68 & 0.50 & 0.34 & 83.3 \\
\hline $\mathrm{Be}$ & 0.66 & 0.44 & 0.40 & 79.8 \\
\hline Cs & 0.64 & 0.58 & 0.05 & 74.8 \\
\hline As & 0.52 & 0.67 & -0.12 & 74.0 \\
\hline W & 0.52 & 0.75 & -0.04 & 83.6 \\
\hline $\mathrm{Ba}$ & 0.50 & 0.64 & 0.32 & 75.8 \\
\hline $\mathrm{Ag}$ & 0.48 & 0.75 & 0.03 & 79.9 \\
\hline $\mathrm{Cu}$ & 0.42 & 0.65 & -0.01 & 60.8 \\
\hline $\mathrm{Tl}$ & 0.39 & 0.76 & 0.24 & 78.6 \\
\hline $\mathrm{Zn}$ & 0.36 & 0.84 & -0.03 & 84.3 \\
\hline $\mathrm{Sb}$ & 0.33 & 0.80 & -0.08 & 75.5 \\
\hline Mo & 0.31 & 0.76 & 0.25 & 73.0 \\
\hline In & 0.29 & 0.83 & 0.23 & 83.0 \\
\hline $\mathrm{Cd}$ & 0.22 & 0.86 & 0.09 & 79.3 \\
\hline $\mathrm{Te}$ & 0.21 & 0.83 & 0.20 & 77.5 \\
\hline $\mathrm{Bi}$ & 0.08 & 0.84 & 0.34 & 81.6 \\
\hline $\mathrm{Pb}$ & 0.05 & 0.89 & -0.05 & 79.4 \\
\hline I & 0.49 & 0.22 & 0.66 & 72.3 \\
\hline Sc & 0.38 & 0.19 & 0.78 & 79.4 \\
\hline $\mathrm{Br}$ & -0.01 & 0.02 & 0.90 & 81.5 \\
\hline $\mathrm{K}$ & -0.25 & 0.14 & 0.90 & 89.6 \\
\hline Total variability (\%) & 41.9 & 28.4 & 9.9 & 80.2 \\
\hline Eingene value & 24.3 & 4.48 & 3.27 & \\
\hline
\end{tabular}

Bolded values represent dominant factor loadings for each factor. 
$3400 \mathrm{mg} / \mathrm{kg}$ in the sediment of the Kamenička River and $930 \mathrm{mg} / \mathrm{kg}$ in the fluvisol.

Although the geographical distribution of the microelements in alluvial soils is closely related to the composition of the parent materials, the soils often exhibit less variation in micronutrient contents than their parent rocks. Soil-forming processes greatly influence the trace element distribution within the soil profile. $^{[12]}$ Significant variations were not detected in the contents of the other elements in traces in the sediment and in the fluvisol soils, as well as in relation to the Dutch standards for these elements. Considering the rest of the elements, the overview of the contents of microelements and elements in traces by zones is provided in Table 4 .

Student T-tests and F-tests were used to determine the significant differences in the contents of each element among the zones along the course of the river Bregalnica (Table 4). The first two zones in the upper course of the river showed significant statistical difference only in relation to the following elements: $\mathrm{Al}, \mathrm{Ca}, \mathrm{Br}, \mathrm{K}, \mathrm{Pb}, \mathrm{Sb}$, and $\mathrm{Te}$ (Table 4). For the rest of the elements, there were insignificant distribution variations. This area (Zones 1 and 2) lithologically relies on dominance of the Quaternary alluvium. The second zone, in comparison with the third zone, which starts after the mouth of the Zletovska River, that is, after the Kalimanci hydro accumulation, showed significant differences for almost all of the analyzed elements, except for $\mathrm{As}, \mathrm{Co}, \mathrm{Cu}, \mathrm{Eu}, \mathrm{Fe}, \mathrm{Mg}$ and $\mathrm{Na}$ (Table 4). Between zones 2 and 3 there is a separate lithological unit, which shows predominance of Proterosoic granite, shales and gneisses (Fig. 3). The comparison of all elements between the third and the fourth zone pointed to significant statistical differences in the contents of all the 69 elements analyzed.

The comparative analysis of the different zones in the area of the Bregalnica river basin showed that the whole basin cannot be defined as a single area. There are certain lithogenic and anthropogenic factors that condition the distribution of different chemical elements, which differs from the expected natural distribution. The environmental disaster that took place in the Kamenička River (2003) because of discharge of flotation tailings is clearly confirmed today by the high contents of lead and zinc in the sediment and in the alluvial soils. These high metal contents are introduced with the river water into the waters of the hydro accumulation Kalimanci, which serves as anthropogenic filter for the high contents of lead and zinc that can reach the waters of the river Bregalnica.

\section{Multivariate assessment for the dominant geochemical association}

Apart from the monitoring of the distribution of the different chemical elements, that is, establishing their contents in the sediment and in the hydromorphic soils on the river terraces, the correlations of the 69 analyzed elements were also statistically processed. For this reason, based on factor analysis, three synthetic geochemical associations of elements were singled out. The first two geochemical associations are dominant factors with variability of $41.9 \%$ and $28.4 \%$ out of the total variability of the factor analysis (Table 5). In order to confirm the

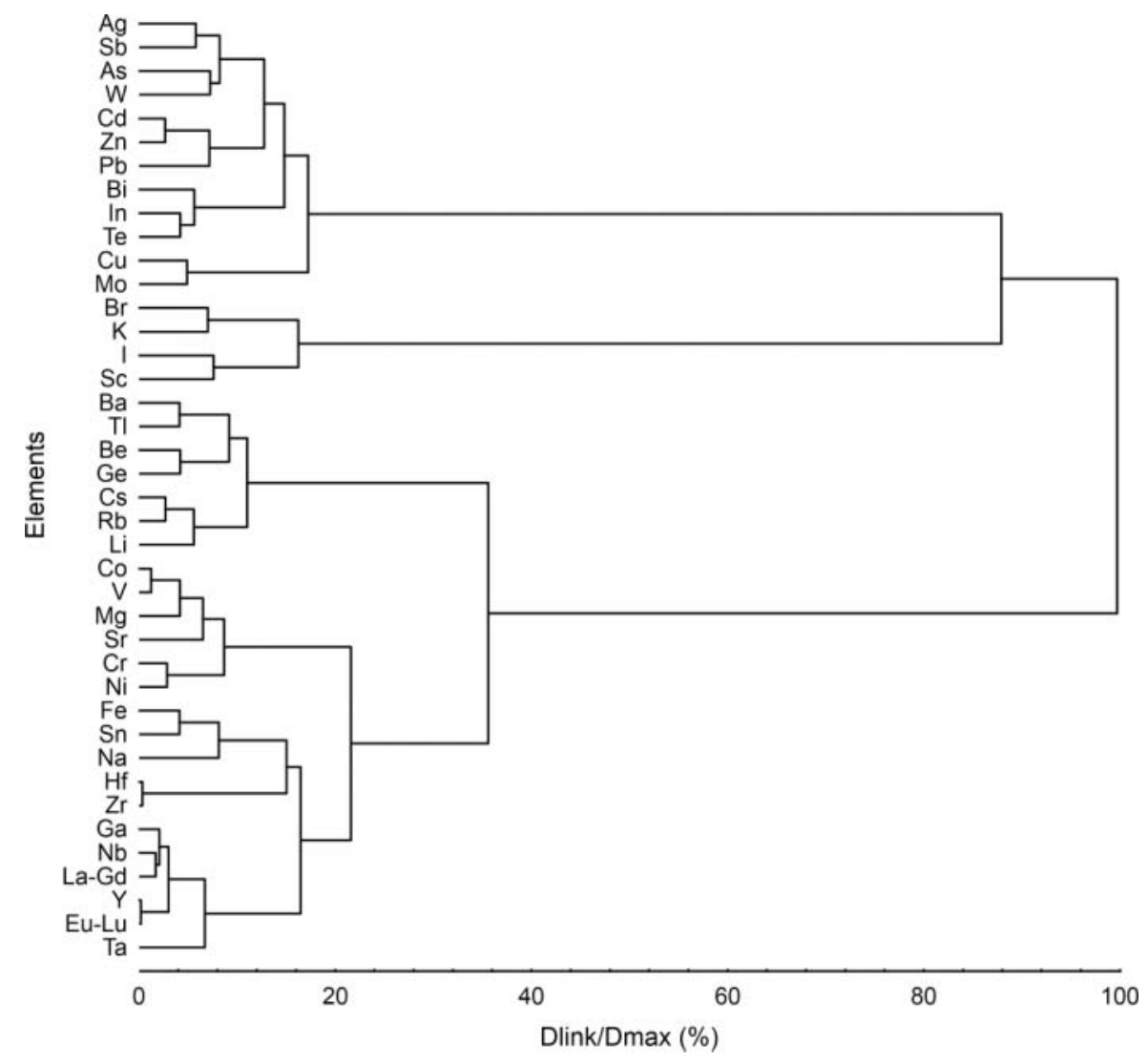

Figure 5. Dendrogram of dominant multivariate associations. 


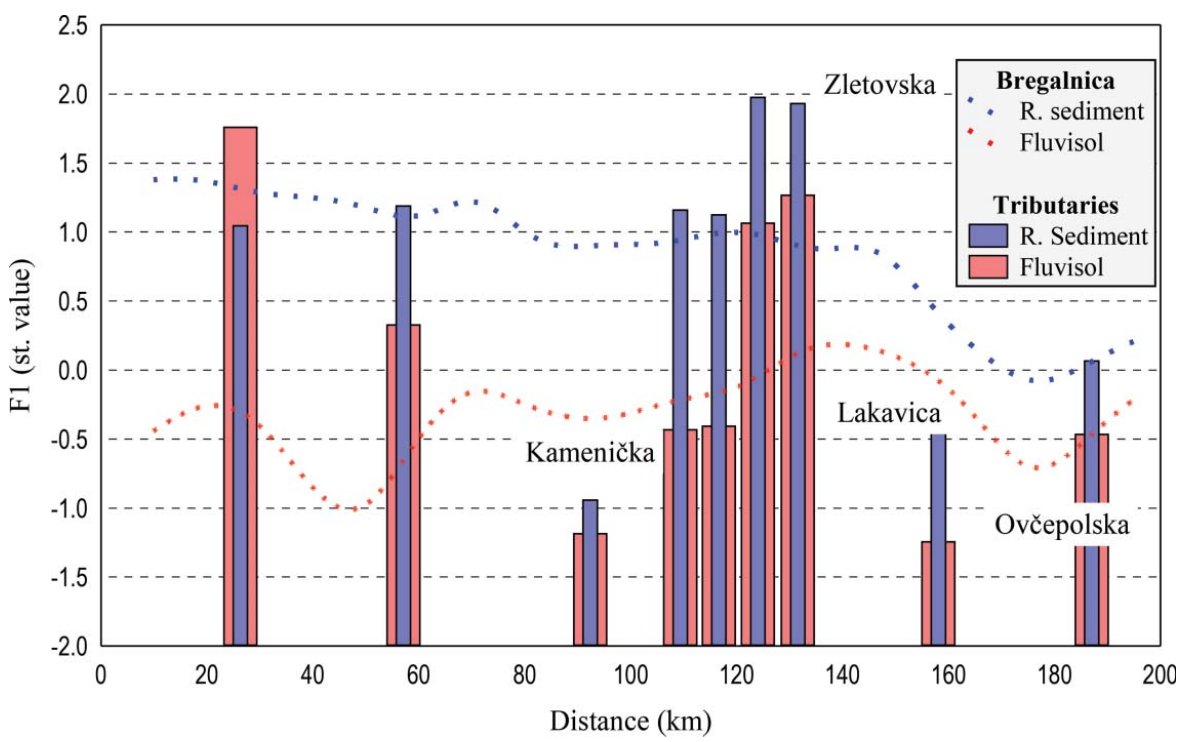

Figure 6. Distribution of F1 elements: Y-Eu-Lu-Cr-V-La-Gd-Nb-Co-Hf-Zr-Ga-Mg-Fe-Sr-Ta-Sn-Li-Na-Rb-Ni-Ge-Be-Cs in the sediment and the fluvisol soils in the course of the Bregalnica river and its tributaries. Tributaries: Ratevska River, Ochepalska River, Kamenička River, Osojnica, Orizarska River, Kočanska River, Zletovska River, Lakavica, Ovčepolska River.

inter-correlation of the elements contents, a cluster dendogram was also constructed (Fig. 5).

Factor 1 (F1) represents a natural geochemical association of the elements: Y-Eu-Lu-Cr-V-La-Gd-Nb-Co-Hf-Zr-Ga-MgFe-Sr-Ta-Sn-Li-Na-Rb-Ni-Ge-Be-Cs. As a dominant factor with Eingen-value of significance of 24.3, it points to a stable distribution and strong correlation among these lithogenic elements. Their contents are due to the long-term deposition of the particles driven by the river water, that is, the particles created due to the earth erosion processes, rain-wash of rocks, particles carried by the wind etc. This dominant geochemical association is not subject to certain anthropogenic influences, which is also confirmed by the association of the rare earth elements (REEs) Eu-Lu and La-Gd. REE in terrigenous grains are largely unreactive and contribute an inherited signature. ${ }^{[30]}$ The role of sediments as carriers and potential sources of
REEs and metals is well established and a number of studies on the river basin and estuaries have yielded fundamental features of the aquatic geochemistry of the REEs. ${ }^{[30,31]}$ Rare earth elements in the sedimentary rocks are mostly terrigenous and reveal the source rock composition reflecting the REE distribution in the exposed continental crust. These studies indicate that chemical-weathering reactions on the continent lead to extensive fractionation between the dissolved REEs and the river suspended particles and continental rocks. ${ }^{[15]}$ Fractionation of REEs and metals occur predominantly in the solid phase during transport in the river systems. This geochemical association indicates very good stability in distribution along the whole course of the river.

Factor 2 (F2) is a geochemical association, which is processed based on the significant correlations among the elements: As, W, Ba, Ag, Cu, Tl, Zn, Sb, Mo, In, Cd, Te, Bi,

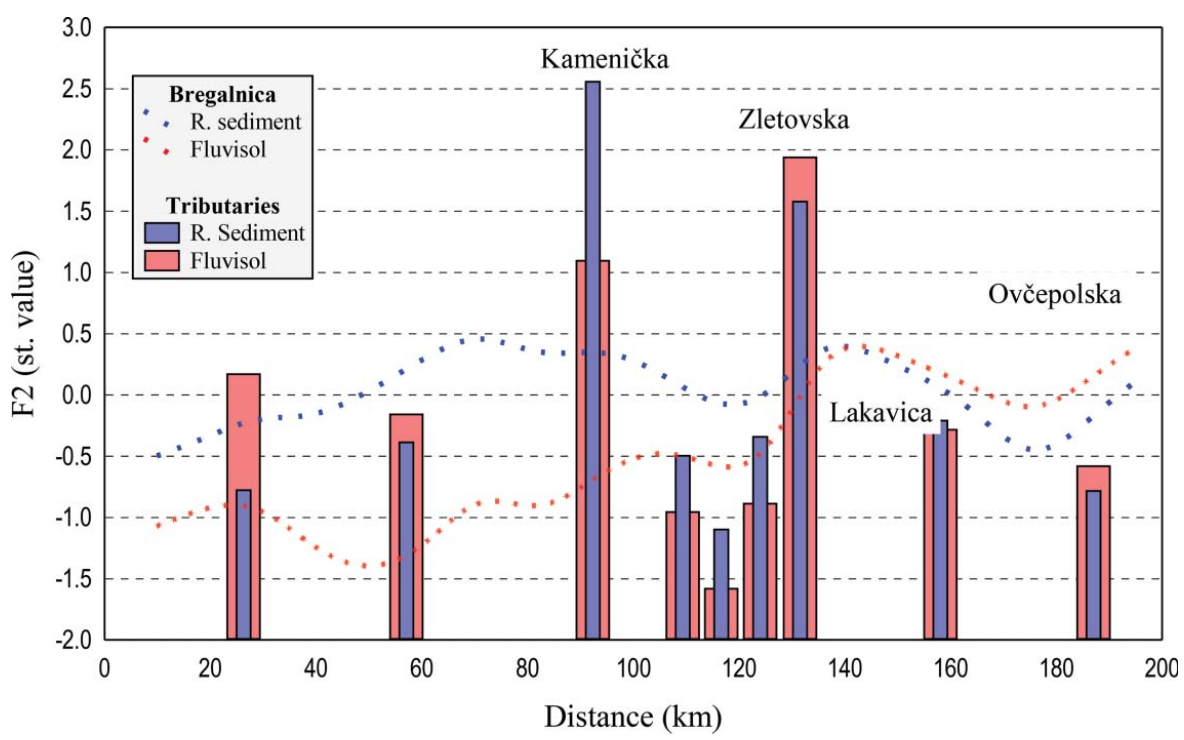

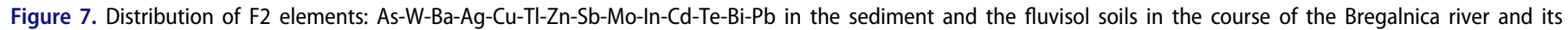
tributaries. Tributaries: Ratevska River, Ochepalska River, Kamenička River, Osojnica, Orizarska River, Kočanska River, Zletovska River, Lakavica, Ovčepolska River. 
and $\mathrm{Pb}$. The contents of these elements are very frequently concentrated as a result of the processing of mineral raw materials. The association of $\mathrm{Cd}, \mathrm{Pb}, \mathrm{Zn}, \mathrm{Cu}, \mathrm{Sb}$ and $\mathrm{Mo}$ confirms this fact because the $90^{\text {th }}$ percentile areas of the distribution of these elements associate in similar areas. An enhanced mobilisation of metals as dissolved organic complexes was observed for $\mathrm{Pb}, \mathrm{Cu}$ and $\mathrm{Zn}{ }^{[32]}$ and for $\mathrm{Cu}$ and As, but not for $\mathrm{Cd}$ and $\mathrm{Zn} .{ }^{[33]}$ Charlatchka and Cambier found that $\mathrm{Pb}$ was especially complexed by organic acids in the alluvial soil. ${ }^{[34]}$ The enrichment of these elements is dominant in Zone 3 where there is a significant use of the land for agricultural purposes. Application of technical measures leads to a significant increase in the organic mass in this area, and consequently to the increase in the mobility of $\mathrm{As}, \mathrm{Cu}, \mathrm{Pb}$ and $\mathrm{Zn}$ in this area.

Factor 3 (F3) is the least distinguished factor with variance of $9.9 \%$ out of the total variance and E-value of $3.3 \%$. This geochemical association of elements is with non-specific characterization of distribution. The dominant elements are $\mathrm{Br}$ and $\mathrm{K}$ with values for factor loadings of 0.90 . The

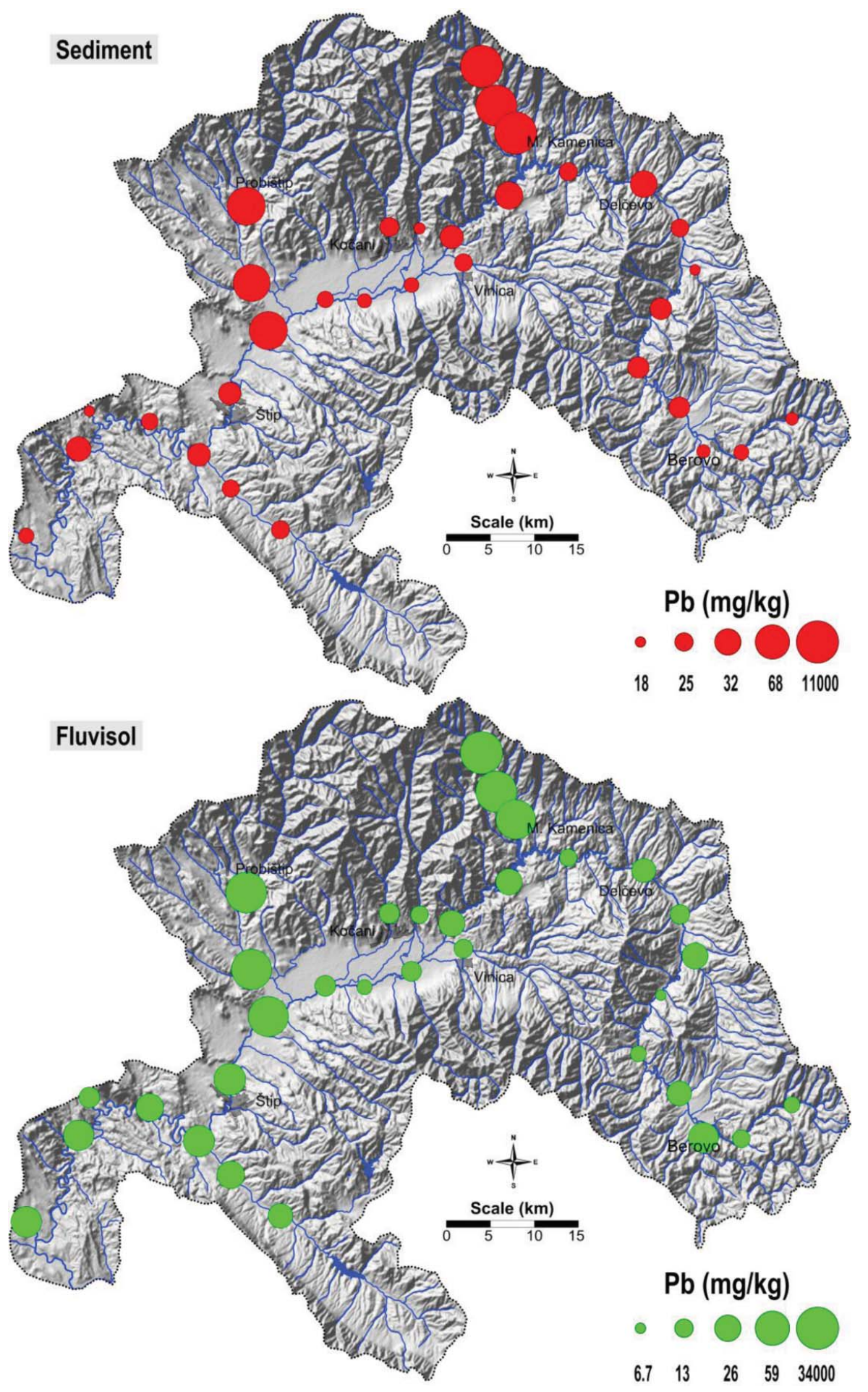

Figure 8. Spatial distribution of lead in the sediment and the fluvisol soil in the Bregalnica river basin. 
contents of these elements are also primarily conditioned by the morphological profile of the alluvium. The halogen elements show affinity among each other also in the soil lithogenic structures, on which this association is probably based. There is certain specific trend of enrichment of these elements in Zone 1, where Quaternary alluvium predominates (Fig. 3). This factor is closely correlated with the distribution of the second factor (As-W-Ba-Ag-Cu-Tl-Zn-Sb-Mo-In-Cd$\mathrm{Te}-\mathrm{Bi}-\mathrm{Pb}$ ), i.e. the occurrence of the oldest volcanic rocks has significant influence on both Factors 2 and 3, which clearly points to their inter-correlation, even though they are isolated as separate factors (Fig. 5).

\section{Areal distribution of the geochemical dominant associations}

The two dominant geochemical associations are also presented graphically using bar plots, for enhanced visual overview of the distribution. The distribution of the elements of the first

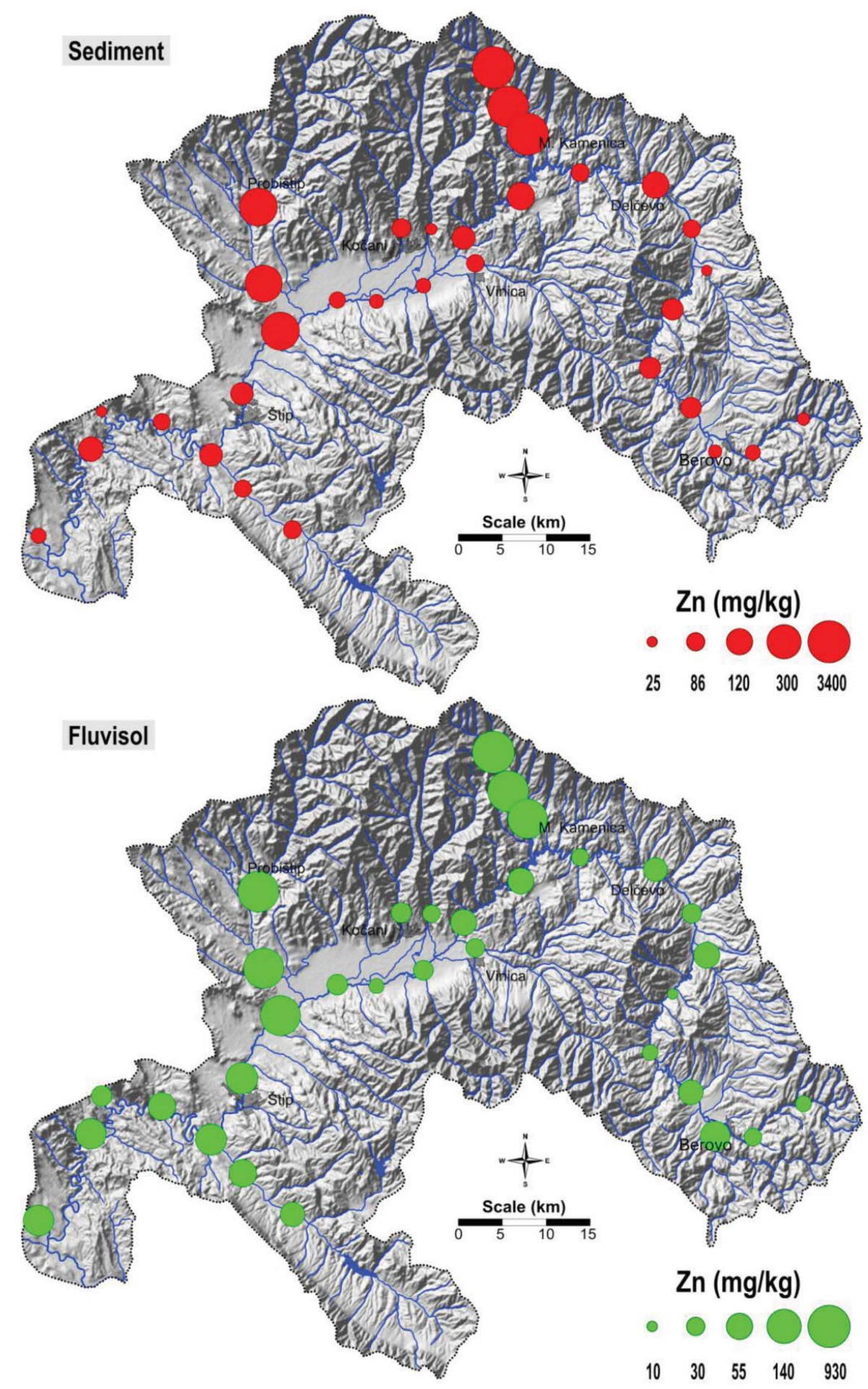

Figure 9. Spatial distribution of zinc in the sediment and the fluvisol soil in the Bregalnica river basin. 
geochemical association (F1) in sediments and fluvisol soils is presented in Figure 6. The contents of these elements are almost constant along the whole course of the Bregalnica river both in the sediment and on the river terraces (fluvisol soils). The contents of these elements behave in a similar way in the tributaries, with lower presence in the Kamenicka River, Lakavica and the Ovčepolska River (Fig. 6). Usually, sediments are significantly more enriched with lithogenic elements compared to the fluvisol. ${ }^{[2,6,35,36]}$ The geochemistry of large rivers will provide insights to the erosional processes of the study region on a global scale due to the differential mobility taking place between the metals by denudation. ${ }^{[37,38]}$ Water-rock interaction, especially at low temperature is unlikely to cause substantial change in REE distribution in all sediments. Hence the REEs are insoluble with extremely low concentrations in dissolved form in the river and are mainly transported and deposited as detrital materials in the river basins.

The second geochemical association (F2) behaves very similarly to the first as regards the contents of the elements in the sediments and fluvisol soils. Along the whole course of the Bregalnica river a certain continuous trend of the contents of these elements is perceived, with a mild increase in the area after the mouth of the Zletovska River. Unlike Factor 1, the distribution of the second geochemical association in the sediment and the fluvisol soils of the tributaries is significantly more distinguished. Maximum contents of this factor are detected in the sediment of the Kamenička River, and, with a very mild decrease, in the Zletovska River. Cd-Pd-Zn-Sb stand out as dominant variables in this association. High contents of lead and zinc are found both in the sediment and the fluvisol. The pollution of the Kamenička River is alleviated by the presence of the hydro accumulation Kalimanci, which, on its part, acts as an accumulator of the high contents and deposits of the flotation tailings from the mine Sasa. The Zletovska River behaves similarly to the Kamenička River, with high contents of the elements of this geochemical association, with particular emphasis on lead and zinc (Fig. 7).

The anthropogenic introduction of $\mathrm{Pb}$ and $\mathrm{Zn}$ is due to the continuous outpour of wastewaters from the ore and the flotation plants. These deposits are carried by the river water, which introduces them into the river Bregalnica. In this way, the Zletovska River is a more intensively polluted than the Kamenička River, although the maximum contents of lead and zinc are found in the sediment and the alluvium of the Kamenička River. For better visual representation, spatial distribution maps for lead and zinc in the whole investigated areas are provided (Figs. 8 and 9). The sediment represents a natural archive for the geochemistry of the elements. The historical record for the geochemistry of $\mathrm{Pb}$ inputs in the river shows significant dominance of $\mathrm{Pb}$ in the sediment vs. fluvisol (Fig. 8). This is particularly evident in the content of lead in Zone 3 where the median of this element is significantly reduced in the fluvisol, which is not the case for the sediment. This phenomenon is even more dominant in determining the total Zn content (Fig. 9).

\section{Conclusion}

The focus of this paper is on the mapping of the lithogenic and anthropogenic distribution of a total of 69 elements in alluvial soil samples from the Bregalnica river basin. The specific polymetallic enrichments occur in the areas of the very old Neogene volcanism (Makedonska Kamenica, Zone 3) and Oligocene volcanism (Kratovo-Zletovo district, Zone 4). Strong relations occur between these dominant lithological units and the hydrothermal exploitation in the area of the $\mathrm{Pb}-\mathrm{Zn}$ Sasa mine, the $\mathrm{Pb}-\mathrm{Zn}$ Zletovo mine and the Bučim copper mine.

Dominant geochemical association of the following elements was presented: Y-Eu-Lu-Cr-V-La-Gd-Nb-Co-Hf-Zr-Ga-Mg-Fe$\mathrm{Sr}-\mathrm{Ta}-\mathrm{Sn}-\mathrm{Li}-\mathrm{Na}-\mathrm{Rb}-\mathrm{Ni}-\mathrm{Ge}-\mathrm{Be}-\mathrm{Cs}$. This geochemical marker is related to the dominance of Quaternary alluvium along the upper course of the river Bregalnica. The distribution patterns of these metals mainly reflect the lithological control of the drainage basin. This finding is also supported by the abundance of $\mathrm{Al}$, $\mathrm{Fe}$ and $\mathrm{Mn}$ in the river sediments and the fluvisol. The second dominant geochemical association, As-W-Ba-Ag-Cu-Tl-Zn-Sb$\mathrm{Mo}-\mathrm{In}-\mathrm{Cd}-\mathrm{Te}-\mathrm{Bi}-\mathrm{Pb}$, indicates enrichment of certain elements in the areas of mineral $\mathrm{Pb}-\mathrm{Zn}$ deposits. This may be attributed partly to the weak soil development and lack of soil contamination in the studied zones. On the contrary, $\mathrm{Pb}$ and $\mathrm{Zn}$ contents are mainly determined by the pedophytogenic controls rather than the lithogenic factors.

The distribution of trace elements within the individual zones showed a relationship to some soil-forming processes (with emphasis on $\mathrm{Zn}$ content). $\mathrm{Pb}$ contents were strongly correlated with the hydrothermal exploitations especially in the area of very old volcanism (Neogene clastites). The areas of Neogene vulcanites were characterized with the natural enrichments of $\mathrm{Fe}$ and $\mathrm{Cd}$, due to the poly-metallic unused area (Berovo-Vladimirovo, poly-metallic anomaly, Zone 1).

This study suggests that the further investigations should be focused on: a) determining the geochemical interaction of the elements between soil horizons, separately in the areas of Neogene volcanism (Sasa mine) vs. Oligocene volcanism (KratovoZletovo); b) quantification of the enrichment factors for the trace elements in topsoil along the Bregalnica river basin, hunting the geochemical markers for this unique geological area.

\section{Funding}

This work was funded by Core Program, under the support of ANCSI, Project No. PN16.40.02.01.

\section{References}

[1] Burton, A.G.; Baudo, R.; Beltrami, M.; Rowland, C. Assessing sediment contamination using six toxicity assays. J. Limnol. 2001, 60, 263-267.

[2] Birch, G.; Taylor, S.; Matthai, C. Small-scale spatial and temporal variance in the concentration of heavy metals in aquatic sediments: a review and some new concepts. Environ. Pollut. 2001, 113, 357-372.

[3] Zahra, A.; Hashmi, M.Z.; Malik, R.N.; Ahmed, Z. Enrichment and geo-accumulation of heavy metals and risk assessment of sediments of the Kurang Nallah-feeding tributary of the Rawal Lake Reservoir, Pakistan. Sci. Total Environ. 2013, 470-471, 925-933.

[4] Zhang, C.; Yu, Z.G.; Zeng, G.M.; Jiang, M.; Yang, Z.Z.; Cui, F.; Hu, L. Effects of sediment geochemical properties on heavy metal bioavailability. Environ. Int. 2014, 73, 270-281.

[5] Santos Bermejo, J.; Beltrán, R.; Gómez Ariza, J. Spatial variations of heavy metals contamination in sediments from Odiel river (Southwest Spain). Environ. Int. 2003, 29, 69-77. 
[6] Sterckeman, T.; Douay, F.; Baize, D.; Fourrier, H.; Proix, N.; Schvartz, C. Trace element in soils developed in sedimentary materials Northern France. Geoderma. 2006, 136, 912-929.

[7] Gopinath, A.; Nair, S.M.; Kumar, N.C.; Jayalakshmi, K.V.; Pamalal, D. A baseline study of trace metals in a coral reef sedimentary environment, Lakshadweep Archipelago. Environ. Earth Sci. 2009, 59, 1245-1266.

[8] Nobi, E.P.; Dilipan, E.; Thangaradjou, T.; Sivakumar, K.; Kannan, L. Geochemical and geostatistical assessment of heavy metal concentration in the sediments of different coastal ecosystems of Andaman Islands, India. Estuar. Coast. Shelf Sci. 2010, 87, 253-264.

[9] Yi, Y.; Yang, Z.; Zhang, S. Ecological risk assessment of heavy metals in sediment and human health risk assessment of heavy metals in fishes in the middle and lower reaches of the Yangtze River basin. Environ. Pollut. 2011, 159, 2575-2585.

[10] Du Laing, G.; Rinklebe, J.; Vandecasteele, B.; Meers, E.; Tack, F. M.G. Trace metal behaviour in estuarine and riverine floodplain soils and sediments: a review. Sci. Total Environ. 2009, 407(13), 3972-3985.

[11] Lin, Y.C.; Chang-Chien, G.P.; Chiang, P.C.; Chen, W.H.; Lin, Y.C. Multivariate analysis of heavy metal contaminations in seawater and sediments from a heavily industrialized harbor in Southern Taiwan. Mar. Pollut. Bull. 2013, 76, 266-275.

[12] Nael, M.; Khademi, H.; Jalalian, A.; Schulin, R.; Kalbasi, M.; Sotohian, F. Effect of geopedological conditions on the distribution and chemical speciation of selected trace elements in forest soils of western Albaroz, Iran. Geoderma. 2009, 152, 157-170.

[13] Arunachalam, K.D.; Annamalai, S.K.; Baskaran, K.V.; Rajaram, S.; Jha, S.K. Spatial and multivariate analysis of trace elements in the surface water and deep sediments of fresh water aquatic ecosystem. Am. J. Environ. Sci. 2014, 10(2), 102-122.

[14] Stafilov, T. Environmental pollution with heavy metals in the Republic of Macedonia. Contribution, Section of Natural, Mathematical and Biotechnical Sciences, MASA 2014, 35(2), 81-119.

[15] Balabanova, B.; Stafilov, T.; Šajn, R. Litogenic distribution of rare earth elements in automorphic and alluvial soils from Bregalnica river basin. Maced. J. Chem. Chem. En. 2015, 34(1), 201-212.

[16] Stafilov, T.; Balabanova, B.; Šajn, R. Geochemical atlas of the region of the Bregalnica river basin, Faculty of Natural Sciences and Mathematics: Skopje, 2014.

[17] Lazarevski, A. Climate in Macedonia, Kultura: Skopje, 1993.

[18] Barcikowski, J.; von Quadt, A.; Gallhofer, D.; Peytcheva, I.; Heinrich, C.A.; Serafimovski, T. Geochronology, geochemistry and isotope tracing of the Oligocene magmatism of the Buchim-Damjan-Borov Dol ore district: Implications for timing, duration and source of the magmatism. Lithos. 2013, 180, 216-233.

[19] Dumurdzanov, N.; Serafimovski, T.; Burchfiel, B.C. Evolution of the Neogene-Pleistocene Basins of Macedonia. Geological Society of America, Digital Map and Chart Series 1: 2004.

[20] Rakićević, T.; Stojanov, P.; Arsovski, M. Basic Geological Map of SFRJ, Sheet Prilep, M 1:100,000 (map \& interpreter), Federal Geological Survey: Beograd, 1965.

[21] Serafimovski, T.; Tasev, G.; Dolenec, T. Petrological and geochemical features of the Neogene volcanites of the Osogovo Mountains, eastern Macedonia. RMZ-M\&G. 2006, 52(3), 523-534.

[22] Serafimovski, T.; Stefanova, V.; Volkovb, A.V. Dwarf copper-gold porphyry deposits of the Buchim-Damjan Borov Dol Ore District,
Republic of Macedonia (FYROM). Geol. Ore Deposit. 2010, 52(3), 179-195.

[23] Cassard, D.; Bertrand, G.; Maldan, F.; Gaàl, G.; Juha, K.; Aatos, S.; et al. ProMine pan-European Mineral Deposit database: a new dataset for assessing primary mineral resources in Europe. Mineral Resources Potential Maps: a Tool for Discovering Future Deposits, 2012.

[24] Lehmann, St.; Barcikowski, J.; von Quadt, A.; Gallhofer, D.; Peytcheva, I.; Heinrich, C.A.; Serafimovski, T. Geochronology, geochemistry and isotope tracing of the Oligocene magmatism of the Buchim-DamjanBorov Dol ore district: Implications for timing, duration and source of the magmatism. Lithos. 2013, 180-181, 216-233.

[25] Alderton, D.H.M.; Serafimovski, T.; Mullen, B.; Fairall, K.; James, S. The chemistry of waters associated with metal mining in Macedonia. Mine Water Environ. 2005, 24(3), 139-149.

[26] ISO 14869-1:2001. Soil quality-Dissolution for the determination of total element content-Part 1: Dissolution with hydrofluoric and perchloric acids. International Organization for Standardization: Switzerland, Geneva, 2001.

[27] Box, G.E.P.; Cox, D.R. An analysis of transformations. J.R. Stat. Soc. Series B Methodology. 1964, 26(2), 211-252.

[28] Šajn, R. Factor analysis of soil and attic-dust to separate mining and metallurgy influence, Meza Valley, Slovenia. Math. Geol. 2006, 38 , 735-747.

[29] Žibret, G.; Šajn, R. Hunting for geochemical associations of elements: factor analysis and self-organizing maps. Math. Geol. 2010, 42, 681703.

[30] Ramesh, R.; Ramanathan, A.L.; Arthur James, R.; Subramanian, V.; Jacobsen, S.B.; Holland, H.D. Rare earth elements and heavy metal distribution in estuarine sediments of east coast of India. Hydrobiologia. 1999, 57, 1-11.

[31] Ramesh, R.; Ramanathan, A.L.; Ramesh, S.; Purvaja, R.; Subramanian, V. Distribution of rare earth elements in the surficial sediments of the Himalaya river system. Geochem. J. 2000, 34, 295-319.

[32] Alvim Ferraz, M.C.M.; Lourenço, J.C.N. The influence of organic matter content of contaminated soils on the leaching rate of heavy metals. Environ. Prog. 2000, 19, 53-58.

[33] Kalbitz, K.; Wennrich, R. Mobilization of heavy metals and arsenic in polluted wetland soils and its dependence on dissolved organic matter. Sci. Total Environ. 1998, 209, 27-39.

[34] Charlatchka, R.; Cambier, P. Influence of reducing conditions on solubility of trace metals in contaminated soils. Water Air Soil Pollut. 2000, 118, 143-167.

[35] Alves, R.I.S.; Sampaio, C.F.; Nadal, M.; Schuhmacher, M.; Domingo, J.L.; Segura-Muñoz, S.I. Metal concentrations in surface water and sediments from Pardo River, Brazil: Human health risks. Environ. Res. 2014, 133, 149-155.

[36] Alijagić, J.; Šajn, R. Distribution of chemical elements in an old metallurgical area, Zenica (Bosnia and Herzegovina). Geoderma. 2011, $162,71-85$

[37] Goovaerts, P. Estimation or simulation of soil properties? An optimization problem with conflicting criteria. Geoderma. 2000, 97, 165186.

[38] Gaur, V.K.; Gupta, S.K.; Pandey, S.D.; Gopal, K.; Misra, V. Distribution of heavy metals in sediment and water of river Gomti. Environ. Monit. Assess. 2005, 102(1-3), 419-433. 